\title{
Rubin's Integral Refinement of the Abelian Stark Conjecture
}

\author{
Cristian D. Popescu
}

To Harold Stark, on his 65th birthday.

\begin{abstract}
This paper is a survey of results obtained by the present author towards proving Rubin's integral version of Stark's conjecture for abelian $L$ functions of arbitrary order of vanishing at the origin. Rubin's conjecture is stated and its links to the classical integral Stark conjecture for $L$-functions of order of vanishing 1 are discussed. A weaker version of Rubin's conjecture formulated by the author in [P4] is also stated and its links to Rubin's conjecture are discussed. Evidence in support of the validity of Rubin's conjecture is provided. A series of applications of Rubin's conjecture to the theory of Euler Systems, groups of special units and Gras-type conjectures are given.
\end{abstract}

\section{Introduction}

In the 1970s and early 1980s, Stark [St] developed a remarkable Galois-equivariant conjectural link between the values at $s=0$ of the first non-vanishing derivatives of the Artin $L$-functions $L_{K / k}(\rho, s)$ associated to a Galois extension $K / k$ of number fields and a certain $\mathbb{Q}[\operatorname{Gal}(K / k)]$-module invariant associated to the group of global units of $K$. Stark's Main Conjecture should be viewed as a vast Galois-equivariant generalization of the unrefined, rational version of Dirichlet's class-number formula

$$
\lim _{s \rightarrow 0} \frac{1}{s^{r}} \zeta_{k}(s) \in \mathbb{Q}^{\times} \cdot R_{k},
$$

in which the zeta function $\zeta_{k}$ is replaced by a Galois-equivariant $L$-function

$$
\Theta_{K / k, S}(s)=\sum_{\rho \in \widehat{G}} L_{K / k, S}(\rho, s) \cdot e_{\check{\rho}},
$$

with values in the center of the group-ring $\mathcal{Z}(\mathbb{C}[\mathrm{Gal}(K / k)])$, the regulator $R_{k}$ is replaced by a Galois-equivariant regulator with values in $\mathcal{Z}(\mathbb{C}[\mathrm{Gal}(K / k)])$, and the

1991 Mathematics Subject Classification. 11R42, 11R58, 11R27.

Key words and phrases. global $L$-functions, Units, Class Groups, Euler Systems.

The author was supported in part by NSF Grants DMS-0200543 and DMS-0350441. 
rank $r$ of the group of units in $k$ is replaced by the local rank function of the (projective) $\mathbb{Q}[\operatorname{Gal}(K / k)]$-module $\mathbb{Q} U_{S}$ of $S$-units in $K$.

In the 1970s and early 1980s, work of Stark, Tate, and Chinburg among others revealed not only the depth and importance of Stark's Main Conjecture for number theory (e.g. Chinburg's theory of multiplicative Galois Module Structure emerged in this context), but also the fact that an integral refinement of this statement, in the spirit of the integral Dirichlet class-number formula

$$
\lim _{s \rightarrow 0} \frac{1}{s^{r}} \zeta_{k}(s)=-\frac{h_{k}}{w_{k}} \cdot R_{k},
$$

would have very far reaching applications to major unsolved problems in the field. In [St IV], Stark himself formulated such an integral refinement for abelian extensions $K / k$ and their associated imprimitive $L$-functions $L_{K / k, S}(\chi, s)$ of order of vanishing at most 1 at $s=0$. Roughly speaking, this integral statement predicts the existence of a special $S$-unit $\varepsilon_{K}$ in $K$, which has remarkable arithmetic properties and, if evaluated against the Galois-equivariant regulator, it produces the value at $s=0$ of the first derivative of the Galois-equivariant $L$-function $\Theta_{K / k, S}(s)$. Moreover, $\varepsilon_{K}$ is unique and computable in terms of these special $L$-values.

In all the major instances in which this conjecture has been proved, the Stark units $\varepsilon_{K}$ have turned out to be truly remarkable arithmetic objects. They are cyclotomic $S$-units or Gauss sums if $k=\mathbb{Q}$ (see [St IV] and [Ta4]), elliptic units if $k=\mathbb{Q}(\sqrt{-d})$, with $d \in \mathbb{Z}_{\geq 1}$ (see [St IV] and [Ta4]), and norms of torsion points of sign-normalized rank 1 Drinfeld modules if $k$ is a function field (see [H1]). In each of these instances the construction of Stark's units $\varepsilon_{K}$ is closely related to the solution of Hilbert's 12th problem for the respective base field $k$ (i.e. the explicit generation of the abelian class-fields of the field $k$.) Tate [Ta4] showed that this is not at all coincidental - it turns out that, when non-trivial, the solution to Stark's Integral Conjecture would lead to an explicit generation of the abelian class-fields of the base field $k$ by exponentials of special values of $L$-functions.

In the late 1980s and early 1990s, the emergence of Kolyvagin's theory of Euler Systems revealed a new interpretation of the known Stark units $\varepsilon_{K}$ - they provide us with the only known non-trivial examples of Euler Systems of units: the Euler Systems of cyclotomic units, Gauss sums, elliptic units, and torsion points of rank 1 Drinfeld modules, leading to enlightening solutions of major problems in number theory - the Iwasawa Main Conjecture over $\mathbb{Q}$ and over quadratic imaginary fields, particular cases of the Birch-Swinnerton Dyer conjecture etc. Rubin [Ru2] showed that this is not a coincidence either - it turns out that, when non-trivial, the Stark units $\varepsilon_{K}$ for various extensions $K / k$ give rise to Euler Systems over a fixed field $k$.

Unfortunately, since in most cases the order of vanishing at $s=0$ of the nonprimitive $L$-functions $L_{K / k, S}(\chi, s)$ is strictly larger than 1, Stark's integral refinement of his Main Conjecture has a non-trivial output $\varepsilon_{K}$ for a very limited class of abelian extensions $K / k$. This is why an integral refinement of Stark's Main Conjecture in its full generality is needed. Such a refinement is also expected to have the type of applications described in the previous paragraph for general extensions $K / k$. In 1994, Rubin [Ru3] formulated an integral refinement of the Main Conjecture for abelian extensions $K / k$ and their associated imprimitive $L$-functions $L_{K / k, S}(\chi, s)$ of arbitrary order of vanishing at $s=0$.

The main objectives of this paper are as follows: after introducing the necessary notations and definitions ( $\$ 1$ below), we state Rubin's Conjecture and a related 
refinement of Stark's Main Conjecture due to the present author and discuss their links to the classical Integral Stark Conjecture ( 22$)$; we provide evidence in support of the validity of Rubin's Conjecture ( $\S 3$ ); finally, we discuss a series of applications of Rubin's Conjecture to the theory of Euler Systems, the construction of groups of special units and Gras-type conjectures $(\S 4)$. We do not include applications of Rubin's Conjecture to Hilbert's 12th problem, nor do we discuss our recent $\ell$-adic refinements of Rubin's statement and their links to well known conjectures of Gross [Gro1-3], postponing the treatment of these (otherwise) important topics for other occasions.

This introduction would be incomplete without mentioning the recent remarkable work of Burns, Flach, and their students and collaborators on the Equivariant Tamagawa Number Conjecture (ETNC), which has brought a wide variety of new and exciting ideas, techniques, and interpretations to the subject of Stark's Conjectures. Building upon earlier work of Bloch-Kato and Fontaine-Perrin-Riou, Burns and Flach [BF1-3] have formulated the ETNC for $L$-functions associated to motives with not necessarily abelian coefficients. If restricted to Artin motives, this statement can also be viewed as an integral refinement of Stark's Main Conjecture for general (not necessarily abelian) extensions $K / k$. In the more restrictive case of Dirichlet motives, the ETNC is closely related to Rubin's Conjecture, as shown by Burns in [Bu1]. We refer the reader to Flach's contribution to this volume and Burns's upcoming papers for more details in this direction.

We dedicate this paper to Harold Stark, on the occasion of his 65th birthday, with admiration and gratitude for his outstanding work, which has already inspired several generations of number theorists and will continue to do so for many more generations to come.

\section{Notations and definitions}

1.1. Notations. Let $K / k$ be a finite, abelian extension of global fields of arbitrary characteristic and of Galois group $G:=\operatorname{Gal}(K / k)$. We denote by $\widehat{G}$ the group of irreducible complex valued characters of $G$. Let $\mu_{K}$ be the group of roots of unity in $K, w_{K}:=\operatorname{card}\left(\mu_{K}\right)$, and $S$ and $T$ two finite, nonempty sets of primes in $k$. For a finite extension $K^{\prime} / k, S_{K^{\prime}}$ and $T_{K^{\prime}}$ will denote the sets of primes in $K^{\prime}$ dividing primes in $S$ and $T$, respectively . For the moment, we require that the sets $S$ and $T$ satisfy the following set of hypotheses.

\section{Hypotheses $\left(\mathbf{H}_{0}\right)$}

1. $S$ contains all the primes which ramify in $K / k$, and all the infinite primes of $k$ in the case where $k$ is a number field.

2. $T \cap S=\emptyset$.

3. There are no nontrivial elements in $\mu_{K}$ which are congruent to 1 modulo all the primes $w$ in $T_{K}$.

The reader will note that the last hypothesis above is automatically satisfied in the function field case. In the number field case, it is satisfied if, for example, $T$ contains either at least two primes of different residual characteristic or a prime whose corresponding residue field is large compared to the size of $\mu_{K}$.

For a finite extension $K^{\prime} / k, O_{K^{\prime}, S}$ will denote its ring of $S_{K^{\prime}}$-integers, $U_{K^{\prime}, S}:=$ $O_{K^{\prime}, S}^{\times}$is the group of $S_{K^{\prime}}$-units in $K^{\prime}$, and $A_{K^{\prime}, S}$ the ideal-class group of $O_{K^{\prime}, S}$. For any such $K^{\prime}$, we also define the $(S, T)$-modified group of units and respectively 
ideal class-group as follows.

$$
\begin{aligned}
U_{K^{\prime}, S, T} & :=\left\{x \in U_{K^{\prime}, S} \mid x \equiv 1 \bmod w, \forall w \in T_{K^{\prime}}\right\} . \\
A_{K^{\prime}, S, T} & :=\frac{\left\{\text { fractional ideals of } O_{K^{\prime}, S} \text { coprime to } T_{K^{\prime}}\right\}}{\left\{x \cdot O_{K^{\prime}, S} \mid x \equiv 1 \bmod w, \forall w \in T_{K^{\prime}}\right\}} .
\end{aligned}
$$

For simplicity, we will set $U_{S}:=U_{K, S}, A_{S}:=A_{K, S}, U_{S, T}:=U_{K, S, T}$, and $A_{S, T}:=$ $A_{K, S, T}$. Since $S_{K}$ and $T_{K}$ are $G$-invariant, these groups are endowed with natural $\mathbb{Z}[G]$-module structures.

1.2. The $G$-equivariant $L$-function. For $K / k, S$, and $T$ as above, and any $\chi$ in $\widehat{G}$, let $L_{S}(\chi, s)$ denote, as usual, the $L$-function associated to $\chi$ with Euler factors at primes in $S$ removed, of the complex variable $s$. This is a complex valued function, holomorphic everywhere if $\chi$ is non-trivial, and holomorphic outside $s=1$, with a pole of order 1 at $s=1$ if $\chi$ is the trivial character. With the help of these $L$-functions, one can define

$$
\begin{gathered}
\Theta_{S}, \quad \Theta_{S, T}: \mathbb{C} \longrightarrow \mathbb{C}[G], \\
\Theta_{S}(s):=\sum_{\chi \in \widehat{G}} L_{S}(\chi, s) \cdot e_{\chi^{-1}}, \quad \Theta_{S, T}:=\prod_{v \in T}\left(1-\sigma_{v}^{-1} \cdot(\mathrm{N} v)^{1-s}\right) \cdot \Theta_{S}(s),
\end{gathered}
$$

where $\sigma_{v}$ and $\mathrm{N} v$ denote the Frobenius morphism in $G$ and the cardinality of the residue field associated to $v$, and

$$
e_{\chi^{-1}}:=1 /|G| \sum_{\sigma \in G} \chi(\sigma) \cdot \sigma
$$

is the idempotent element associated to $\chi^{-1}$ in $\mathbb{C}[G]$. The functions $\Theta_{S}$ and $\Theta_{S, T}$ are the so-called $S$-modified respectively $(S, T)$-modified $G$-equivariant $L$-function. If the group-ring $\mathbb{C}[G]$ is viewed in the obvious manner as a direct product of $|G|$ copies of $\mathbb{C}$, then the projections of $\Theta_{S}$ and $\Theta_{S, T}$ onto the various components of $\mathbb{C}[G]$ with respect to this product decomposition are holomorphic away from $s=1$ (holomorphic everywhere, respectively) as functions of the complex variable $s$. The values of $\Theta_{S, T}$ at non-positive integers satisfy the following remarkable integrality property.

TheOREM 1.2.1. Under hypotheses $\left(H_{0}\right)$, one has

$$
\Theta_{S, T}(1-n) \in \mathbb{Z}[G],
$$

for all integers $n \geq 1$.

In the number field case, Theorem 1.2.1 was independently proved by Deligne-Ribet $[\mathbf{D R}]$, P. Cassou-Nogues $[\mathbf{C N}]$, and D. Barski $[\mathbf{B a r} \mathbf{1}]$. In fact, in $[\mathbf{D R}]$ it is proved that one has

$$
\operatorname{Ann}_{\mathbb{Z}[G]}\left(\mu_{K}^{\otimes n}\right) \cdot\left(\sum_{\chi \in \widehat{G}} L_{S}(\chi, 1-n) \cdot e_{\chi^{-1}}\right) \subseteq \mathbb{Z}[G] \text {, for all } n \in \mathbb{Z}_{\geq 1} .
$$

However, the reader will notice right away that hypothesis $\left(\mathrm{H}_{0}\right) 3$ is equivalent to the statement

$$
\prod_{v \in T}\left(1-\sigma_{v}^{-1} \cdot(\mathrm{N} v)^{n}\right) \in \operatorname{Ann}_{\mathbb{Z}[G]}\left(\mu_{K}^{\otimes n}\right), \text { for all } n \in \mathbb{Z}_{\geq 1}
$$


In fact, the following lemma, whose proof in characteristic 0 can be found in $[\mathbf{C o}]$, holds true in both characteristics 0 and $p$.

Lemma 1.2.2. Assume that the set of data $\left(K / k, S_{0}\right)$ satisfies hypothesis $\left(\mathrm{H}_{0}\right)$ 1. Then, for all $n \in \mathbb{Z}_{\geq 1}, \operatorname{Ann}_{\mathbb{Z}[G]}\left(\mu_{K}^{\otimes n}\right)$ is generated as a $\mathbb{Z}[G]$-module by the elements

$$
\delta_{T}(n):=\prod_{v \in T}\left(1-\sigma_{v}^{-1} \cdot(N v)^{n}\right),
$$

where $T$ runs through all the finite sets of primes in $k$, such that $(K / k, S, T)$ satisfies hypotheses $\left(\mathrm{H}_{0}\right)$.

In the function field case, Theorem 1.2.1 is a direct consequence of Weil's theorem expressing the $L$-functions as alternating products of characteristic polynomials of the action of a geometric Frobenius morphism on various $G$-eigenspaces of $\ell$-adic étale cohomology groups of the smooth projective curve associated to the top field $K$. By using this interpretation, one can show that, if $q$ is the cardinality of the field of constants for the base field $k$, then there exists a polynomial $P_{S, T}(X)$ in $\mathbb{Z}[G][X]$, such that

$$
\Theta_{S, T}(s)=P_{S, T}\left(q^{-s}\right)
$$

(see [Ta4] and $\S 3.1$ below.) Theorem 1.2.1 follows immediately from this equality. In what follows, in order to simplify notations we will set

$$
\delta_{T}:=\delta_{T}(1)
$$

for any set $T$ as in Lemma 1.2.2.

In the context described above, Stark's Conjecture provides a link between the lead term in the Taylor expansion at $s=0$ of the $G$-equivariant $L$-function $\Theta_{S, T}(s)$ and certain arithmetic invariants of the abelian extension $K / k$. In order to make this link precise, we will need to impose additional conditions on the sets of primes $S$ and $T$. Let us fix an integer $r \geq 0$. We associate to $r$ the following (extended) set of hypotheses to be satisfied by the set of data $(K / k, S, T, r)$.

\section{Hypotheses $\left(\mathbf{H}_{r}\right)$}

1. $S$ contains all the primes which ramify in $K / k$, and all the infinite primes of $k$ in the case where $k$ is a number field.

2. $\operatorname{card}(S) \geq r+1$.

3. $S$ contains at least $r$ distinct primes which split completely in $K / k$.

4. $T \cap S=\emptyset$.

5. There are no nontrivial elements in $\mu_{K}$ which are congruent to 1 modulo all the primes $w$ in $T_{K}$.

The following Lemma shows how hypotheses $\left(\mathrm{H}_{r}\right)$ control the order of vanishing $\operatorname{ord}_{s=0}$ at $s=0$ of the associated $G$-equivariant $L$-functions.

LEMma 1.2.3. If $(K / k, S, T)$ satisfies hypotheses $\left(H_{r}\right)$, then $\operatorname{ord}_{s=0} L_{S}(\chi, s) \geq$ $r, \forall \chi \in \widehat{G}$ and, consequently,

$$
\operatorname{ord}_{s=0} \Theta_{S}(s)=\operatorname{ord}_{s=0} \Theta_{S, T}(s) \geq r .
$$


Proof. This is a direct consequence of the following equality proved in [Ta4].

$$
\operatorname{ord}_{s=0} L_{S}(\chi, s)=\left\{\begin{array}{ll}
\operatorname{card}\left\{v \in S|\chi|_{G_{v}}=\mathbf{1}_{G_{v}}\right\}, & \text { for } \chi \neq \mathbf{1}_{G} \\
\operatorname{card}(S)-1, & \text { for } \chi=\mathbf{1}_{G}
\end{array},\right.
$$

where $G_{v}$ is the decomposition group of $v$ in $K / k$ and $\mathbf{1}_{H}$ denotes the trivial character of a group $H$.

If the set of data $(K / k, S, T)$ satisfies hypotheses $\left(\mathrm{H}_{r}\right)$, we let

$$
\Theta_{S, T}^{(r)}(0):=\lim _{s \rightarrow 0} \frac{1}{s^{r}} \Theta_{S, T}(s)
$$

denote the coefficient of $s^{r}$ in the Taylor expansion of $\Theta_{S, T}(s)$ at $s=0$.

1.3. The $G$-equivariant regulator maps. The link between the analytic aspects of the picture (represented by the $G$-equivariant $L$-function described in the previous section) and its arithmetic aspects (represented by the $\mathbb{Z}[G]$-modules of units $U_{S, T}$ and ideal classes $A_{S, T}$ ) predicted by Stark's Conjecture is achieved via certain $G$-equivariant regulator maps. These will be defined in the present section.

Throughout this section we assume that the data $(K / k, S, T, r)$ satisfies hypotheses $\left(\mathrm{H}_{r}\right)$, for a fixed $r \in \mathbb{Z}_{\geq 0}$. We fix an $r$-tuple $V:=\left(v_{1}, \ldots, v_{r}\right)$ of $r$ distinct primes in $S$ which split completely in $K / k$, and primes $w_{i}$ in $K$, with $w_{i}$ dividing $v_{i}$, for all $i=1, \ldots, r$. Let $W:=\left(w_{1}, \ldots, w_{r}\right)$. For all primes $w$ in $K$, let $|\cdot|_{w}$ denote their associated metrics, canonically normalized so that the product formula holds. This means that for all $x \in K^{\times}$, we let

$$
|x|_{w}:=\left\{\begin{array}{ll}
(\mathrm{N} w)^{-\operatorname{ord}_{w}(x)}, & \text { if } w \text { is finite } \\
\left|\sigma_{w}(x)\right|, & \text { if } w \text { is infinite }
\end{array},\right.
$$

where $\sigma_{w}$ denotes the unique embedding of $K$ into $\mathbb{C}$ associated to $w$ and $|\cdot|$ is the usual complex absolute value.

Throughout this paper, if $M$ is a $\mathbb{Z}[G]-$ module and $R$ is a commutative ring with 1 , then $R M$ denotes the tensor product $R \otimes_{\mathbb{Z}} M$ endowed with the usual $R[G]$-module structure, $\widetilde{M}$ denotes the image of $M$ via the canonical morphism

$$
M \longrightarrow \mathbb{Q} M
$$

and $M^{*}:=\operatorname{Hom}_{\mathbb{Z}[G]}(M, \mathbb{Z}[G])$ is the dual of $M$ in the category of $\mathbb{Z}[G]-$ modules.

Definition 1.3.1. The $G$-equivariant regulator map associated to $W$ is the unique $\mathbb{Q}[G]$-linear morphism

$$
R_{W}: \mathbb{Q} \bigwedge_{\mathbb{Z}[G]}^{r} U_{S, T} \longrightarrow \mathbb{C}[G]
$$

such that, for all $u_{1}, \ldots, u_{r}$ in $U_{S, T}$, we have

$$
R_{W}\left(u_{1} \wedge \cdots \wedge u_{r}\right):=\operatorname{det}\left(-\sum_{\sigma \in G} \log \left|u_{i}^{\sigma^{-1}}\right|_{w_{j}} \cdot \sigma\right),
$$

where the determinant is taken over $\mathbb{C}[G]$, and $i, j=1, \ldots, r$. 
REMARK 1. If extended by $\mathbb{C}$-linearity the regulator $R_{W}$ defined above induces a $\mathbb{C}[G]$-isomorphism (see $[\mathbf{R u} 3]$ for a proof)

$$
R_{W}: \mathbb{C} \stackrel{r}{\mathbb{Z}[G]}_{\hat{C}_{S, T}}^{\sim} U_{S} \stackrel{\sim}{\longrightarrow}[G],
$$

also denoted by $R_{W}$ in what follows. By definition, the special value $\Theta_{S, T}^{(r)}(0)$ belongs to the $\mathbb{C}[G]$-submodule of $\mathbb{C}[G]$, consisting of all elements $x \in \mathbb{C}[G]$ which satisfy

$$
e_{\chi} \cdot x=0,
$$

for all $\chi$ in $\widehat{G}$, such that $\operatorname{ord}_{s=0} L_{S}(\chi, s)>r$. Since $R_{W}$ is a $\mathbb{C}[G]$-isomorphism, this implies that there exists a unique element

$$
\varepsilon_{S, T} \in \mathbb{C} \underset{\mathbb{Z}[G]}{\stackrel{r}{\wedge}} U_{S, T},
$$

such that the following equalities hold.

1. $R_{W}\left(\varepsilon_{S, T}\right)=\Theta_{S, T}^{(r)}(0)$.

2. $e_{\chi} \cdot \varepsilon_{S, T}=0$ in $\underset{\mathbb{Z}[G]}{\stackrel{r}{r}} U_{S, T}$, for all $\chi$ in $\widehat{G}$, such that $\operatorname{ord}_{s=0} L_{S}(\chi, s)>r$.

The above remark prompts us to give the following definition.

Definition 1.3.2. Assume that the set of data $(K / k, S, T, r)$ satisfies hypotheses $\left(H_{r}\right)$. Let $M$ be a $\mathbb{Z}[G]$-module. We define

$\widetilde{M}_{r, S}:=\left\{x \in \widetilde{M} \mid e_{\chi} \cdot x=0\right.$ in $\mathbb{C} M$, for all $\chi \in \widehat{G}$ such that $\left.\operatorname{ord}_{s=0} L_{S}(\chi, s)>r\right\}$.

\section{The Conjectures}

In this section, we state Stark's Conjecture ("over $\mathbb{Q}$ ") in the abelian situation outlined in $\S 1$, as well as its integral refinements ("over $\mathbb{Z}$ ") due to Rubin and the present author.

\subsection{Stark's Conjecture "over $\mathbb{Q}$ ".}

CONJECTURE A $(K / k, S, T, r)$ (STARK). If the set of data $(K / k, S, T, r)$ satisfies hypotheses $\left(\mathrm{H}_{r}\right)$, then there exists a unique element

$$
\varepsilon_{S, T} \in \mathbb{Q} \underset{\mathbb{Z}[G]}{\stackrel{r}{\wedge}} U_{S, T},
$$

such that the following equalities hold.

1. $R_{W}\left(\varepsilon_{S, T}\right)=\Theta_{S, T}^{(r)}(0)$.

2. $e_{\chi} \cdot \varepsilon_{S, T}=0$ in $\mathbb{C} \underset{\mathbb{Z}[G]}{\stackrel{r}{\wedge}} U_{S, T}$, for all $\chi$ in $\widehat{G}$, such that $\operatorname{ord}_{s=0} L_{S}(\chi, s)>r$.

A few remarks concerning this statement are in order.

REMARK 1 . The uniqueness part of the statement above is a direct consequence of Remark $1, \S 1.3$. The (highly non-trivial) conjectural part is the statement that the unique element $\varepsilon_{S, T} \in\left(\mathbb{C} \underset{\mathbb{Z}[G]}{\stackrel{r}{\wedge}} U_{S, T}\right)_{r, S}$ satisfying the regulator condition (1) and the vanishing condition $(2)$ in Remark $1, \S 1.3$, belongs in fact to the $\mathbb{Q}[G]$ submodule $\left(\mathbb{Q} \underset{\mathbb{Z}[G]}{\stackrel{r}{r}} U_{S, T}\right)_{r, S}$. 
REMARK 2. No doubt, the reader has noticed that the notation used for $\varepsilon_{S, T}$ does not capture its dependence of our choice of $r$-tuples $V:=\left(v_{1}, \ldots, v_{r}\right)$ and $W=\left(w_{1}, \ldots, w_{r}\right)$ of primes in $S$ and $S_{K}$, as in $\S 1.3$. It is indeed true that $\varepsilon_{S, T}$ depends on these choices. However, it turns out that this dependence is simple and, most importantly, if Conjecture A is true for one choice of $V$ and $W$, then it is true for any other choice. To show this, let us first assume that $V^{\prime}$ and $W^{\prime}$ differ from $V$ and $W$ by a permutation $\tau \in \operatorname{Sym}_{r}$ and respectively an $r$-tuple $\sigma:=\left(\sigma_{1}, \ldots, \sigma_{r}\right) \in G^{r}$, i.e. $V^{\prime}=\left(v_{\tau(1)}, \ldots, v_{\tau(r)}\right)$ and $W^{\prime}=\left(v_{\tau(1)}^{\sigma_{1}}, \ldots, v_{\tau(r)}^{\sigma_{r}}\right)$. Then one can define a unique $\mathbb{Z}[G]$-linear isomorphism

$$
\Phi_{\tau, \sigma}: \underset{\mathbb{Z}[G]}{\stackrel{r}{\wedge}} U_{S, T} \stackrel{\sim}{\longrightarrow} \underset{\mathbb{Z}[G]}{\stackrel{r}{\wedge}} U_{S, T}
$$

by setting $\Phi_{\tau, \sigma}\left(u_{1} \wedge \cdots \wedge u_{r}\right):=u_{\tau(1)}^{\sigma_{1}^{-1}} \wedge \cdots \wedge u_{\tau(r)}^{\sigma_{r}^{-1}}$, for all $u_{1}, \ldots, u_{r}$ in $U_{S, T}$. Let $\Phi_{\tau, \sigma}$ denote the unique $\mathbb{C}$-linear extension of the above map to $\underset{\mathbb{Z}[G]}{\stackrel{r}{\wedge}} U_{S, T}$. If $\varepsilon_{S, T}$ and $\varepsilon_{S, T}^{\prime}$ are the unique elements in $\underset{\mathbb{C}}{\mathbb{R}[G]} \stackrel{r}{\wedge} U_{S, T}$ satisfying (1) and (2) in Remark 1, $\S 1.3$, for $R_{W}$ and $R_{W^{\prime}}$ respectively, then one can easily show that $\varepsilon_{S, T}^{\prime}=\Phi_{\tau, \sigma}\left(\varepsilon_{S, T}\right)$.

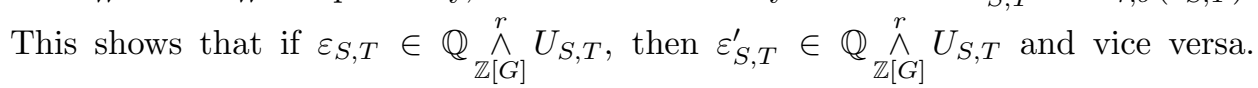
Secondly, let us assume that $S$ contains more than $r$ primes which split completely, i.e. there are at least two choices $V$ and $V^{\prime}$ which do not differ from one another via a permutation $\tau \in \operatorname{Sym}_{r}$. If $\operatorname{card}(S)>r+1$, then Lemma 1.2 .3 above implies that $\Theta_{S, T}^{(r)}(0)=0$ and therefore Conjecture A is trivially true for $\varepsilon_{S, T}=0$, for any choice of $V$ and $W$. Now, let us assume that $S=\left\{v_{1}, \ldots, v_{r+1}\right\}$, and $v_{i}$ splits completely for all $i=1, \ldots, r+1$. Then Lemma 1.2.3 combined with the $(S, T)$-class-number formula of $[\mathbf{R u} 3]$ implies that

$$
\Theta_{S, T}^{(r)}(0)=-\operatorname{card}\left(A_{k, S, T}\right) \cdot R_{k, S, T} \cdot e_{\mathbf{1}_{G}},
$$

where $\mathbf{1}_{G}$ denotes the trivial character of $G$ and $R_{k, S, T}$ is the Dirichlet regulator associated to the group of $(S, T)$-units $U_{k, S, T}$. The equalities above imply right away that in this case Conjecture $\mathrm{A}$ is true with

$$
\varepsilon_{S, T}:= \pm \frac{\operatorname{card}\left(A_{k, S, T}\right)}{|G|^{r}} u_{1} \wedge \cdots \wedge u_{r}
$$

where $u_{1}, \ldots, u_{r}$ is a $\mathbb{Z}$-basis for $U_{k, S, T}$ and the sign is uniquely determined by the choice of $V$ and $W$. Based on these considerations and in order to simplify notations, we have dropped the dependence of $\varepsilon_{S, T}$ on the choice of $V$ and $W$ from the notation in the statement of Conjecture A and throughout this paper.

REmark 3. The reader familiar with [St] will notice that Stark's original formulation of his conjecture "over $\mathbb{Q}$ " is quite different from ours. The main difference stems from the fact that while the statement above is given in a Galois-equivariant form, Stark's original conjecture was formulated in a character-by-character manner (i.e. for one $L$-function at a time). Yet another difference is marked by the fact that Stark deals with all the characters $\chi \in \widehat{G}$, while Conjecture A only deals with those characters $\chi$ whose associated $L$-functions have minimal order of vanishing $r$ at $s=0$. The statement presented here is essentially due to Tate [Ta4] and $\mathrm{Ru}$ bin $[\mathbf{R u 3}]$ and, under the present hypotheses $\left(\mathrm{H}_{r}\right)$, is equivalent to Stark's original 
conjecture for $L$-functions of minimal order of vanishing $r$ at $s=0$ (see [Ru3 $]$ for a proof).

2.2. Rubin's integral refinement of Conjecture A. In this section, we state Rubin's integral refinement of Conjecture A. The main idea behind any integral refinement of Conjecture $\mathrm{A}$ is to construct an arithmetically meaningful $\mathbb{Z}[G]-$ submodule (i.e. a $G$-equivariant lattice of rank which is not necessarily maximal) of the $\mathbb{Q}[G]$-module $\mathbb{Q} \bigwedge_{\mathbb{Z}[G]}^{r} U_{S, T}$, which contains the element $\varepsilon_{S, T}$. We will first describe Rubin's construction of such a lattice. We are still working under hypotheses $\left(\mathrm{H}_{r}\right)$ for the set of data $(K / k, S, T, r)$. For any $(r-1)$-tuple

$$
\Phi:=\left(\phi_{1}, \ldots, \phi_{r-1}\right) \in\left(U_{S, T}^{*}\right)^{r-1}
$$

there exists a unique $\mathbb{Q}[G]$-linear morphism

$$
\widetilde{\Phi}: \mathbb{Q} \bigwedge_{\mathbb{Z}[G]}^{r} U_{S, T} \longrightarrow \mathbb{Q} U_{S, T}
$$

such that, for all $u_{1}, \ldots, u_{r}$ in $U_{S, T}$, we have

$$
\widetilde{\Phi}\left(u_{1} \wedge \cdots \wedge u_{r}\right)=\sum_{k=1}^{r}(-1)^{k} \operatorname{det}\left(\phi_{i}\left(u_{j}\right)\right)_{j \neq k} \cdot u_{j} .
$$

In the last equality, the determinant in the $k$-th term of the sum is taken with respect to all $i=1, \ldots, r-1$ and all $j=1, \ldots, r$, such that $j \neq k$. Please note that since $U_{S, T}$ has no $\mathbb{Z}$-torsion (see hypothesis $\left.\left(\mathrm{H}_{r}\right) 5\right), U_{S, T}$ can be naturally viewed as a $\mathbb{Z}[G]$-submodule of $\mathbb{Q} U_{S, T}$. Consequently, the $k$-term sum above can be viewed without any ambiguity inside $\mathbb{Q} U_{S, T}$.

Definition 2.2.1. Rubin's lattice $\Lambda_{S, T}$ consists of all elements $\epsilon$ in $\underset{\mathbb{Q}}{\mathbb{Z}[G]} \stackrel{r}{\wedge} U_{S, T}$, which satisfy the following properties.

1. $\tilde{\Phi}(\epsilon) \in U_{S, T}$, for all $\Phi:=\left(\phi_{1}, \ldots, \phi_{r-1}\right) \in\left(U_{S, T}^{*}\right)^{r-1}$.

2. $e_{\chi} \cdot \epsilon=0$ in $\mathbb{C}[G]$, for all $\chi \in \widehat{G}$, such that $\operatorname{ord}_{s=0} L_{S}(\chi, s)>r$.

REMARK 1. It is immediate from Definition 2.2.1 that for $r=0,1$, we have

$$
\Lambda_{S, T}= \begin{cases}\left(\widetilde{U_{S, T}}\right)_{1, S}, & \text { if } r=1 \\ \mathbb{Z}[G]_{0, S}, & \text { if } r=0\end{cases}
$$

For a general $r \geq 1$, we have inclusions

$$
|G|^{n} \cdot \Lambda_{S, T} \subseteq\left(\widehat{\bigwedge_{\mathbb{Z}[G]} U_{S, T}}\right)_{r, S} \subseteq \Lambda_{S, T}
$$

for sufficiently large positive integers $n$. It is not difficult to show that if $U_{S, T}$ has finite projective dimension over $\mathbb{Z}[G]$, then the second inclusion above is an equality. However, as Rubin shows in [Ru3], the second inclusion above is in general strict. After tensoring with $\mathbb{Z}[1 /|G|]$, we have an equality

$$
\mathbb{Z}[1 /|G|] \Lambda_{S, T}=\left(\mathbb{Z}[1 /|G|] \widehat{\bigwedge_{\mathbb{Z}[G]}} U_{S, T}\right)_{r, S},
$$


which follows directly from the sequence of inclusions above. Also, since $U_{S, T}$ sits inside $U_{S}$ with a finite index, we have equalities

$$
\mathbb{Q} \Lambda_{S, T}=\left(\mathbb{Q} \bigwedge_{\mathbb{Z}[G]}^{r} U_{S, T}\right)_{r, S}=\left(\mathbb{Q} \bigwedge_{\mathbb{Z}[G]}^{r} U_{S}\right)_{r, S} .
$$

CONJECTURE $\mathrm{B}(K / k, S, T, r)$ (RUBIN). If the set of data $(K / k, S, T, r)$ satisfies hypotheses $\left(\mathrm{H}_{r}\right)$, then there exists a unique element $\varepsilon_{S, T} \in \Lambda_{S, T}$, such that

$$
R_{W}\left(\varepsilon_{S, T}\right)=\Theta_{S, T}^{(r)}(0)
$$

REMARK 2. In the case $r=0$ Rubin's conjecture states that $\Theta_{S, T}(0) \in$ $\mathbb{Z}[G]_{0, S}$. This statement is true and follows immediately if one sets $n=1$ in Theorem 1.2.1 and applies equalities (1) above.

Remark 3. In the case $r=1$, Conjecture B is equivalent to Stark's integral refinement of Conjecture A for $L$-functions of order of vanishing 1 at $s=0$, formulated in [St IV]. A proof of this equivalence can be found in [Ta4] and [Ru3] and it is a direct consequence of Remark 1 for $r=1$ and Lemma 2.2.3 below.

At times, it is convenient to drop dependence on the auxiliary set $T$ in conjecture B formulated above. This is why we will sometimes work with the following statement instead.

CONJECTURE B $(K / k, S, r)$. If the set of data $(K / k, S, r)$ satisfies hypotheses $\left(\mathrm{H}_{r}\right) 1-3$, then for all sets $T$ such that the set of data $(K / k, S, T, r)$ satisfies hypotheses $\left(H_{r}\right)$, there exists a unique element $\varepsilon_{S, T} \in \Lambda_{S, T}$, such that

$$
R_{W}\left(\varepsilon_{S, T}\right)=\Theta_{S, T}^{(r)}(0)
$$

The following result proved in $[\mathbf{P 4}]$ (see Proposition 5.3.1) shows that in the above conjecture it is sufficient to work with minimal sets $T$.

TheOREM 2.2.2. Let $(K / k, S, T, r)$ and $\left(K / k, S, T^{\prime}, r\right)$ be two sets of data satisfying hypotheses $\left(\mathrm{H}_{r}\right)$, such that $T \subseteq T^{\prime}$. Then

$$
B(K / k, S, T, r) \Longrightarrow B\left(K / k, S, T^{\prime}, r\right)
$$

In particular, this theorem shows that, in the case where $\operatorname{char}(k)=p>0$, it suffices to prove conjecture $B(K / k, S, T, r)$ for sets $T$ of cardinality 1 .

REMARK 4. In what follows, we will give a somewhat detailed description of the connection between Rubin's Conjecture and the classical conjectures of Brumer and Brumer-Stark. In the 1970s, Brumer stated the following conjecture, as a natural extension of the classical Theorem of Stickelberger for abelian extensions of $\mathbb{Q}$ (see $[\mathbf{C o}]$ ) to abelian extensions of general number fields. The conjecture was later extended to global fields of arbitrary characteristic by Mazur and Tate (see $[\mathbf{T a} 4])$. 
CONJECTURE $\operatorname{Br}\left(K / k, S_{0}\right)$ (BRUMER). Let us assume that $\left(K / k, S_{0}\right)$ satisfies hypothesis $\left(\mathrm{H}_{0}\right) 1$. Then

$$
\operatorname{Ann}_{\mathbb{Z}[G]}\left(\mu_{K}\right) \cdot \Theta_{S_{0}}(0) \subseteq \begin{cases}\operatorname{Ann}_{\mathbb{Z}[G]}\left(A_{K}\right), & \text { if } \operatorname{char}(k)=0 \\ \operatorname{Ann}_{\mathbb{Z}[G]}\left(\operatorname{Pic}^{0}(K)\right), & \text { if } \operatorname{char}(k)>0\end{cases}
$$

where $A_{K}$ is the usual ideal-class group of the number field $K$ and $\operatorname{Pic}^{0}(K)$ is the Picard group of equivalence classes of divisors of degree 0 of the function field $K$.

The $\mathbb{Z}[G]$-ideal $\operatorname{Ann}_{\mathbb{Z}[G]}\left(\mu_{K}\right) \cdot \Theta_{S_{0}}(0)$ is called the Stickelberger ideal associated to $\left(K / k, S_{0}\right)$ and it generalizes the classical Stickelberger ideal defined for $k=\mathbb{Q}$ to the case of arbitrary base fields $k$. Stark (in the number field case) and Tate (in the function field case) formulated the following refinement of Brumer's conjecture, now commonly known as the Brumer-Stark Conjecture.

CONJECTURE $\operatorname{BrSt}\left(K / k, S_{0}\right)$ (BRUMER-STARK). Let us assume that the set of data $\left(K / k, S_{0}\right)$ satisfies hypothesis $\left(\mathrm{H}_{0}\right) 1$. Then,

1. If $\operatorname{char}(k)=0$ and $I$ is a fractional ideal of $K$, there exists a unique element $\alpha_{I} \in\left(K^{\times}\right)_{0, S_{0}}$, such that

$$
w_{K} \Theta_{S_{0}}(0) \cdot I=\left(\alpha_{I}\right)
$$

and $K\left(\alpha_{I}^{1 / w_{K}}\right) / k$ is an abelian extension.

2. If $\operatorname{char}(k)>0$ and $D$ is a nonzero divisor of $K$, there exist unique $\alpha_{D} \in$ $\left(K^{\times}\right)_{0, S_{0}}$ and $m_{D} \in \mathbb{Z}$, such that

$$
w_{K} \Theta_{S_{0}}(0) \cdot D=\operatorname{div}\left(\alpha_{D}\right)+m_{D} \cdot \sum_{w \in\left(S_{0}\right)_{K}} w
$$

and $K\left(\alpha_{D}^{1 / w_{K}}\right) / k$ is an abelian extension.

Showing that $\operatorname{Br} S t\left(K / k, S_{0}\right)$ implies $\operatorname{Br}\left(K / k, S_{0}\right)$ is an easy exercise based on Lemma 1.2.2 and on the following characterization of those elements $\alpha \in K^{\times}$, such that $K\left(\alpha^{1 / w_{K}}\right) / k$ is an abelian extension (see [Ta4] or [P4]. )

Lemma 2.2.3. Let $\left(K / k, S_{0}\right)$ be as above, let $\alpha \in K^{\times}$, and set

$$
\operatorname{supp}(\alpha):=\left\{v \text { finite prime in } k \mid \operatorname{ord}_{v}\left(N_{K / k}(\alpha)\right)>0\right\} .
$$

Then, the following are equivalent.

1. $K\left(\alpha^{1 / w_{K}}\right) / k$ is an abelian extension.

2. For all finite sets of primes $T$ in $k$, such that $T \cap\left(S_{0} \cup \operatorname{supp}(\alpha)\right)=\emptyset$ and $\left(K / k, S_{0}, T\right)$ satisfies hypotheses $\left(H_{0}\right)$, there exists $\alpha_{T} \in K^{\times}$such that

$$
\alpha^{\delta_{T}}=\alpha_{T}^{w_{K}} ; \quad \alpha_{T} \equiv 1 \bmod ^{\times} w, \text { for all } w \in T_{K} .
$$

This lemma leads us to the following $\left(S_{0}, T\right)$-version of the Brumer-Stark Conjecture, which will be useful in our future considerations.

CONJECTURE $\operatorname{BrSt}\left(K / k, S_{0}, T\right)$. Let us assume that $\left(K / k, S_{0}, T\right)$ satisfies hypothesis $\left(\mathrm{H}_{0}\right)$. Then,

1. If $\operatorname{char}(k)=0$ and $I$ is a fractional ideal of $K$ coprime to $T$, there exists a unique element $\alpha_{I, T} \in\left(K^{\times}\right)_{0, S_{0}}$, such that $\alpha_{I, T} \equiv 1 \bmod ^{\times} w$, for all $w \in$ $T_{K}$, and

$$
\Theta_{S_{0}, T}(0) \cdot I=\left(\alpha_{I, T}\right) .
$$


2. If $\operatorname{char}(k)>0$ and $D$ is a nonzero divisor of $K$ coprime to $T$, there exist unique $\alpha_{D, T} \in\left(K^{\times}\right)_{0, S_{0}}$ and $m_{D} \in \mathbb{Z}$, such that $\alpha_{D, T} \equiv 1 \bmod ^{\times} w$, for all $w \in T_{K}$, and

$$
\Theta_{S_{0}, T}(0) \cdot D=\operatorname{div}\left(\alpha_{D, T}\right)+m_{D} \cdot \sum_{w \in\left(S_{0}\right)_{K}} w
$$

Lemma 2.2.3 implies that proving conjecture $\operatorname{Br} S t\left(K / k, S_{0}\right)$ is equivalent to proving conjectures $\operatorname{Br} S t\left(K / k, S_{0}, T\right)$, for all $T$ such that $\left(K / k, S_{0}, T\right)$ satisfies hypotheses $\left(H_{0}\right)$.

REMARK. The reader will notice right away that, if $\operatorname{char}(k)>0$ and $\operatorname{card}\left(S_{0}\right)>$ 1 , then $\operatorname{BrSt}\left(K / k, S_{0}\right)$ implies that the Stickelberger ideal associated to $\left(K / k, S_{0}\right)$ annihilates in fact the group $\operatorname{Pic}(K)$ of classes of $K$-divisors of arbitrary degree, which is much larger than $\operatorname{Pic}^{0}(K)$. Indeed this can be proved by taking divisor degrees in the equality displayed in Part 2. of the Brumer-Stark Conjecture $\operatorname{Br} S t\left(K / k, S_{0}\right)$ stated above, and by noticing that, if $\operatorname{card}\left(S_{0}\right)>1$, then

$$
\operatorname{deg}\left(w_{K} \Theta_{S_{0}}(0) \cdot D\right)=\mathbf{1}_{G}\left(w_{K} \Theta_{S_{0}}\right) \cdot \operatorname{deg}(D)=0
$$

(see Lemma 1.2.3), for all $K$-divisors $D$. This implies that $m_{D}=0$ and the desired result ensues by applying once again Lemma 1.2.3. If $\operatorname{card}\left(S_{0}\right)=1$, then the Stickelberger ideal does not annihilate $\operatorname{Pic}(K)$, in general.

Rubin's Conjecture fully captures the annihilation phenomenon described in the Brumer-Stark Conjecture, as shown in the following theorem.

THEOREM 2.2.4. Let us assume that the set of data $\left(K / k, S_{0}\right)$ satisfies hypothesis $\left(\mathrm{H}_{0}\right) 1$. Then we have an equivalence

$$
\operatorname{BrSt}\left(K / k, S_{0}\right) \Longleftrightarrow\left\{\begin{array}{l}
B\left(K / k, S_{0} \cup\{v\}, 1\right), \text { for all primes } v \text { in } k, \\
v \text { split in } K / k, v \notin S_{0} .
\end{array}\right\}
$$

Proof (sketch). In the case $\operatorname{char}(k)=0$ this is a consequence of Lemma 2.2.3 above and the fact that each ideal-class in $A_{K}$ has infinitely many representatives $w$, where $w$ is a (finite) prime in $K$, dividing a prime $v$ in $k$ which splits completely in $K / k$ (a consequence of Tchebotarev's density theorem).

In the case $\operatorname{char}(k)>0$ the necessary ingredients for the proof are Lemma 2.2.3 and the fact that the group of $K$-divisor classes $\operatorname{Pic}(K)$ is generated by classes of prime divisors $\Pi$ in $K$ sitting above prime divisors $\pi$ in $k$ which are completely split in $K / k$. (A class-field theoretic proof of this fact can be found in [H1], p. 8.)

2.3. A weaker integral refinement for Conjecture A. In our study of a base-change-type functoriality property for Conjecture B, we arrived at a slightly weaker integral refinement for Conjecture A (see $[\mathbf{P 4}]$ ). From a functorial point of view, this new statement seems to be more natural than Conjecture B. In this section, we state this refinement and describe its links to Conjecture B.

Let us fix $(K / k, S, T, r)$, satisfying hypotheses $\left(\mathrm{H}_{r}\right)$. As in $[\mathbf{P} 4]$, we have an exact sequence of $\mathbb{Z}[G]$-modules

$$
0 \rightarrow U_{S, T} \rightarrow U_{S} \stackrel{\operatorname{res}_{T}}{\longrightarrow} \oplus_{w \in T_{K}} F(w)^{\times} \rightarrow A_{S, T} \rightarrow A_{S} \rightarrow 0,
$$


where $A_{S}$ denotes the ideal class group of the ring of $S$-integers $O_{K, S}$ in $K, F(w)$ is the residue field of $w$, and $\operatorname{res}_{T}$ is the direct sum of the residual morphisms into $F(w)^{\times}$for all $w \in T_{K}$. If we denote by $\mathcal{I}$ the image of $\operatorname{res}_{T}$, and take duals in the category of $\mathbb{Z}[G]$-modules, we obtain the following exact sequence of abelian groups

$$
0 \rightarrow U_{S}^{*} \rightarrow U_{S, T}^{*} \rightarrow \operatorname{Ext}_{\mathbb{Z}[G]}^{1}(\mathcal{I}, \mathbb{Z}[G]) .
$$

Since $\mathcal{I}$ is finite, $\operatorname{Ext}_{\mathbb{Z}[G]}^{1}(\mathcal{I}, \mathbb{Z}[G])$ is finite and consequently $U_{S}^{*}$ can be viewed (via the usual restriction map) as a subgroup of finite (in general non-trivial) index in $U_{S, T}^{*}$. With this observation in mind, one can define a new lattice $\Lambda_{S, T}^{\prime}$ inside

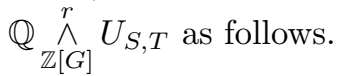

Definition 2.3.1. The lattice $\Lambda_{S, T}^{\prime}$ is the set of all elements $\epsilon$ in $\underset{\mathbb{Q}}{\mathbb{Z}[G]} \stackrel{r}{\wedge} U_{S, T}$, which satisfy the following properties.

1. $\tilde{\Phi}(\epsilon) \in U_{S, T}$, for all $\Phi:=\left(\phi_{1}, \ldots, \phi_{r-1}\right) \in\left(U_{S}^{*}\right)^{r-1}$.

2. $e_{\chi} \cdot \epsilon=0$ in $\mathbb{C}[G]$, for all $\chi \in \widehat{G}$, such that $\operatorname{ord}_{s=0} L_{S}(\chi, s)>r$.

Obviously, since $U_{S}^{*}$ sits inside $U_{S, T}^{*}$ with a finite, in general non-trivial) index, Rubin's lattice $\Lambda_{S, T}$ sits inside the new lattice $\Lambda_{S, T}^{\prime}$ with a finite (in general nontrivial) index. We are now ready to formulate the new integral refinement for Conjecture A mentioned in the introduction.

CONJECTURE C $(K / k, S, r)$. Assume that the set of data $(K / k, S, r)$ satisfies hypotheses $\left(\mathrm{H}_{r}\right) 1-3$. Then, for all sets $T$ such that the set of data $(K / k, S, T, r)$ satisfies hypotheses $\left(\mathrm{H}_{r}\right)$, there exists a unique $\varepsilon_{S, T} \in \Lambda_{S, T}^{\prime}$, such that

$$
R_{W}\left(\varepsilon_{S, T}\right)=\Theta_{S, T}^{(r)}(0) .
$$

Conjecture $\mathrm{C}$ satisfies the following remarkable base-change property. Let us assume that $(K / k, S, 1)$ satisfies hypotheses $\left(\mathrm{H}_{1}\right) 1-3$, and let $k^{\prime}$ be an intermediate field, $k \subseteq k^{\prime} \subseteq K$, and let $r:=\left[k^{\prime}: k\right]$. Then, it is obvious that $\left(K / k^{\prime}, S_{k^{\prime}}, r\right)$ satisfies hypotheses $\left(\mathrm{H}_{r}\right) 1-3$. In [P4] we prove the following.

Theorem 2.3.2 (base-change for Conjecture $\mathrm{C}$ ). If conjecture $C(K / k, S, 1)$ is true, then conjecture $C\left(K / k^{\prime}, S_{k^{\prime}}, r\right)$ is also true.

It would be highly desirable to prove a similar base-change property for Conjecture $\mathrm{B}(K / k, S, r)$. At this time, the techniques developed in [P4] have only led us to showing that, if $(K / k, S, T, 1)$ satisfies hypotheses $\left(\mathrm{H}_{1}\right)$, then

$$
B(K / k, S, T, 1) \Longrightarrow B\left(K / k, S_{k^{\prime}}, T_{k^{\prime}}, r\right) .
$$

This is obviously a much weaker result than the implication

$$
B(K / k, S, r) \Longrightarrow B\left(K / k^{\prime}, S_{k^{\prime}}, r\right),
$$

as the $\operatorname{Gal}\left(k^{\prime} / k\right)$-equivariant sets $T_{k^{\prime}}$ are not minimal in general. However, in $[\mathbf{P} 4]$ we also proved a comparison theorem which links Conjectures B and $\mathrm{C}$ and consequently leads to a base-change result for Conjecture B, if additional requirements are met. In what follows, we briefly describe this result.

If $R$ is a subring of $\mathbb{Q}$ (e.g. $R=\mathbb{Q}, R=\mathbb{Z}[1 /|G|], R=\mathbb{Z}_{(\ell)}$ - the localization of $\mathbb{Z}$ at $\ell$, for $\ell$ prime), then $R B(K / k, S, T, r)$ denotes the statement in Conjecture 
$\mathrm{B}(K / k, S, T, r)$ with the lattice $\Lambda_{S, T}$ replaced by $R \Lambda_{S, T} \subseteq \mathbb{Q} \stackrel{\Upsilon}{\mathbb{R}[G]}_{U_{S, T}}$. We give a similar meaning to $R \mathrm{~B}(K / k, S, r), R \mathrm{C}(K / k, S, r)$, etc. In particular, we have the following equivalences.

(3)

$$
\begin{gathered}
A(K / k, S, T, r) \Longleftrightarrow \mathbb{Q} B(K / k, S, T, r) \Longleftrightarrow \mathbb{Q} B(K / k, S, r) \Longleftrightarrow \mathbb{Q} C(K / k, S, r) \\
B(K / k, S, T, r) \Longleftrightarrow \mathbb{Z}_{(\ell)} B(K / k, S, T, r), \text { for all } \ell \text { prime. }
\end{gathered}
$$

The first equivalence in the top row is a consequence of the last equality in Remark $1, \S 2.2$. The second equivalence in the top row is a consequence of the fact that, for all $T$ as above, the product

$$
\delta_{T}:=\prod_{v \in T}\left(1-\sigma_{v}^{-1} \cdot \mathrm{N} v\right)
$$

is invertible in $\mathbb{Q}[G]$. This implies that, for any $T$ and $T^{\prime}$ such that $(K / k, S, T, r)$ and $\left(K / k, S, T^{\prime}, r\right)$ satisfy hypotheses $\left(\mathrm{H}_{r}\right)$, the unique elements

$$
\varepsilon_{S, T}, \varepsilon_{S, T^{\prime}} \in\left(\mathbb{C} \underset{\mathbb{Z}[G]}{\stackrel{r}{\wedge}} U_{S}\right)_{r, S}
$$

satisfying $R_{W}\left(\varepsilon_{S, T}\right)=\Theta_{S, T}^{(r)}(0)$ and $R_{W}\left(\varepsilon_{S, T^{\prime}}\right)=\Theta_{S, T^{\prime}}^{(r)}(0)$, also satisfy the equality

$$
\varepsilon_{S, T}=\delta_{T} \cdot \delta_{T^{\prime}}^{-1} \cdot \varepsilon_{S, T^{\prime}} .
$$

Therefore, $\varepsilon_{S, T} \in\left(\mathbb{Q} \bigwedge_{\mathbb{Z}[G]}^{r} U_{S}\right)_{r, S}$ if and only if $\varepsilon_{S, T^{\prime}} \in\left(\mathbb{Q} \stackrel{r}{\mathbb{Z}[G]}_{U_{S}}\right)_{r, S}$. Finally, the last equivalence in the top row is a direct consequence of the equality $\mathbb{Q} U_{S}^{*}=\mathbb{Q} U_{S, T}^{*}$.

Let $\mu_{K}$ be the group of roots of unity in $K$, endowed with the usual $\mathbb{Z}[G]-$ module structure. For every prime number $\ell$, let $\mu_{K}^{(\ell)}$ be the $\ell$-Sylow subgroup of $\mu_{K}$. We remind the reader that a $\mathbb{Z}[G]$-module $M$ is called $G$-cohomologically trivial if

$$
\widehat{\mathrm{H}}^{i}(H, M)=0
$$

for all $i \in \mathbb{Z}$ and all subgroups $H \subseteq G$. Here $\widehat{\mathrm{H}}^{i}(H, M)$ denotes the $i$-th Tate cohomology group of $H$ with coefficients in $M$. The following comparison theorem was proved in $[\mathbf{P} \mathbf{4}]$.

THEOREM 2.3.3. Assume that the set of data $(K / k, S, r)$ satisfies hypotheses $\left(H_{r}\right) 1-3$. Then, for all prime numbers $\ell$, the following hold true.

1. $\mathbb{Z}_{(\ell)} B(K / k, S, r) \Longrightarrow Z_{(\ell)} C(K / k, S, r)$.

2. If $\mu_{K}^{(\ell)}$ is $G$-cohomologically trivial or $r=1$, then

$$
\mathbb{Z}_{(\ell)} B(K / k, S, r) \Longleftrightarrow Z_{(\ell)} C(K / k, S, r) .
$$

Since $\mu_{K}$ is $G$-cohomologically trivial if and only if $\mu_{K}^{(\ell)}$ is cohomologically trivial, for all prime numbers $\ell$, and $G$-cohomological triviality is automatic at prime numbers $\ell$ which do not divide $|G|$, Theorem 2.3.3 implies the following.

Corollary 2.3.4. If the set of data $(K / k, S, r)$ satisfies hypotheses $\left(H_{r}\right) 1-3$, then the following hold true.

1. $\mathbb{Z}[1 /|G|] B(K / k, S, r) \Longleftrightarrow \mathbb{Z}[1 /|G|] C(K / k, S, r)$. 
2. If $\mu_{K}$ is $G$-cohomologically trivial or $r=1$, then

$$
B(K / k, S, r) \Longleftrightarrow C(K / k, S, r) .
$$

In particular, Corollary 2.3.4 combined with Theorem 2.3.2 implies the desired base-change property for conjecture $\mathrm{B}(K / k, S, r)$, under the additional hypothesis that $\mu_{K}$ is $G$-cohomologically trivial. The following criterion (see Lemma 5.4.4 in $[\mathbf{P} 4])$ shows that while in the case $\operatorname{char}(k)=0$ cohomological triviality for $\mu_{K}$ is a very rare event, in the case $\operatorname{char}(k)>0$ it is satisfied for very large classes of abelian extensions $K / k$ of a given base field $k$.

LEMMA 2.3.5.

1. If $\ell$ is odd or $\operatorname{char}(k) \neq 0$, then $\mu_{K}^{(\ell)}$ is $G$-cohom. trivial if and only if

$$
\ell \nmid w_{K} \text { or } \ell \nmid\left[K: k\left(\mu_{K}^{(\ell)}\right)\right] .
$$

2. If $\operatorname{char}(k)=0$, then $\mu_{K}^{(2)}$ is $G$-cohom. trivial if and only if

$$
2 \nmid\left[K: k\left(\mu_{K}^{(2)}\right)\right] \text { and }\left\{\begin{array}{l}
k \cap \mathbb{Q}\left(\mu_{K}^{(2)}\right) \text { is not a (totally) real field } \\
\text { in the case } \mu_{K}^{(2)} \neq \mu_{k}^{(2)}
\end{array}\right\} .
$$

In particular, Lemma 2.3.5 implies that, for any odd prime $p$ and $n \geq 1$, $\mathbb{Q}\left(\mu_{p^{n}}\right) / \mathbb{Q}$ satisfies $G$-cohomological triviality for $\mu_{K}^{(\ell)}$ for all odd primes $\ell$ but not for $\ell=2$. On the other hand, if $\operatorname{char}(k)=p>0$ and $K$ is the compositum of an abelian $p$-power degree extension and an arbitrary finite constant field extension of $k$, then the lemma implies that $\mu_{K}$ is $G$-cohomologically trivial (see $\S 3.1$ below). As it turns out, for a given global field $k$ of characteristic $p>0$, the compositum of all the finite abelian extensions $K / k$ obtained this way sits inside the maximal abelian extension $k^{\mathrm{ab}}$ with a "quasi-finite index" (in a sense to be made more precise in $\S 3.1$ below.) Consequently, in characteristic $p>0$, conjectures $\mathrm{B}$ and $\mathrm{C}$ are equivalent for a large class of extensions $K / k$.

\section{Evidence in support of Rubin's Conjecture}

In what follows, we will survey the most significant cases in which Conjecture $B(K / k, S, T, r)$ is known to hold true. Before we begin, we would like to outline the proof strategy adopted in most of these cases. In general, there are two main steps involved.

I. The first step consists of proving Stark's Conjecture "over $\mathbb{Q}$ ", i.e. Conjecture $A(K / k, S, T, r)$, which is equivalent to $\mathbb{Q} B(K / k, S, T, r)$ (see (3) above). This amounts to proving that the unique element $\varepsilon_{S, T} \in\left(\mathbb{C} \wedge_{\mathbb{Z}[G]}^{r} U_{S, T}\right)_{r, S}$ satisfying the regulator condition $R_{W}\left(\varepsilon_{S, T}\right)=\Theta_{S, T}^{(r)}(0)$ belongs in fact to $\left(\mathbb{Q} \wedge_{\mathbb{Z}[G]}^{r} U_{S, T}\right)_{r, S}$.

II. Once step I is completed, it suffices to show that $\varepsilon_{S, T} \in \mathbb{Z}_{(\ell)} \Lambda_{S, T}$, i.e. prove conjecture $\mathbb{Z}_{(\ell)} B(K / k, S, T, r)$, for all prime numbers $\ell$.

In the case where the ordered $r$-tuple $V=\left(v_{1}, \ldots, v_{r}\right)$ of primes in $S$ which split completely in $K / k$ consists only of finite (non-archimedean) primes, then it turns out that for every prime number $\ell$, conjecture $\mathbb{Z}_{(\ell)} B(K / k, S, T, r)$ is a consequence of a strong form of the $\ell$-primary part of the Brumer-Stark Conjecture $\mathbb{Z}_{(\ell)} \operatorname{Br} S t\left(K / k, S_{0}, T\right)$, where $S_{0}:=S \backslash\left\{v_{1}, v_{2}, \ldots, v_{r}\right\}$. So far, the main idea behind 
completing step II has been to prove the strong form of the Brumer-Stark conjecture just mentioned. Below, we formulate this statement and describe its precise link to Rubin's conjecture.

If $R$ is a Noetherian ring and $M$ a finitely generated $R$-module, $\operatorname{Fit}_{R}(M)$ denotes the first Fitting ideal of $M$. For the precise definition and those properties of Fitting ideals relevant in our context, the reader can consult [P2]. The following result is proved in $[\mathbf{R u 3}]$ and $[\mathbf{P 2}]$.

Proposition 3.1. Let $\ell$ be a prime number. Under the above hypotheses, the following implications hold true.

$$
\begin{gathered}
\Theta_{S_{0}, T}(0) \in \mathbb{Z}_{(\ell)}[G]_{0, S_{0}} \cdot \operatorname{Fit}_{\mathbb{Z}_{(\ell)}[G]}\left(A_{S_{0}, T} \otimes \mathbb{Z}_{(\ell)}\right) \Longrightarrow \mathbb{Z}_{(\ell)} B(K / k, S, T, r) . \\
\Theta_{S_{0}, T}(0) \in \mathbb{Z}[G]_{0, S_{0}} \cdot \operatorname{Fit}_{\mathbb{Z}[G]}\left(A_{S_{0}, T}\right) \Longrightarrow B(K / k, S, T, r) .
\end{gathered}
$$

For a set of data $\left(K / k, S_{0}, T\right)$ satisfying hypotheses $\left(\mathrm{H}_{0}\right)$, the statement

$$
\operatorname{SBr} S t\left(K / k, S_{0}, T\right): \Theta_{S_{0}, T}(0) \in \mathbb{Z}[G]_{0, S_{0}} \cdot \mathrm{Fit}_{\mathbb{Z}[G]}\left(A_{S_{0}, T}\right)
$$

will be referred to as the Strong Brumer-Stark conjecture. The reason behind this terminology is the following.

Proposition 3.2. If the set of data $\left(K / k, S_{0}, T\right)$ satisfies hypotheses $\left(H_{0}\right)$ and $\ell$ is any prime number, then

$$
\mathbb{Z}_{(\ell)} \operatorname{SBr} S t\left(K / k, S_{0}, T\right) \Longrightarrow \mathbb{Z}_{(\ell)} \operatorname{Br} S t\left(K / k, S_{0}, T\right)
$$

Proof (sketch). The proof is a direct consequence of the inclusion

$$
\operatorname{Fit}_{R}(M) \subseteq \operatorname{Ann}_{R}(M),
$$

valid for any Noetherian $\operatorname{ring} R$ and finitely generated $R$-module $M$ (see [P2]) and the inclusion

$$
\mathbb{Z}[G]_{0, S_{0}} \subseteq \begin{cases}\cap_{v \in S_{0}} I_{G_{v}}, & \text { if } \operatorname{card}\left(S_{0}\right)>1 \\ \left(\sum_{\sigma \in G / G_{v}} \tilde{\sigma}\right) \mathbb{Z}[G]+I_{G_{v}}, & \text { if } S_{0}=\{v\}\end{cases}
$$

where $I_{G_{v}}$ is the kernel of the canonical ring morphism $\mathbb{Z}[G] \longrightarrow \mathbb{Z}\left[G / G_{v}\right]$, and $\widetilde{\sigma}$ is an inverse image of $\sigma \in G / G_{v}$ under the canonical group morphism $G \longrightarrow G / G_{v}$ (apply Lemma 1.2.3).

So far, in the case where the $r$ split primes in $S$ are finite (non-archimedean), the main line of attack for conjecture $\mathbb{Z}_{(\ell)} B(K / k, S, T, r)$ has consisted of proving the $\ell$-primary part of the Strong Brumer-Stark Conjecture $\mathbb{Z}_{(\ell)} \operatorname{SBr} S t\left(K / k, S_{0}, T\right)$ and then use Proposition 3.1. Unfortunately, as the reader will see below, although this method has been successful in a wide variety of cases (in both characteristics 0 and $p$ ), we cannot hope to rely on it for all the remaining cases, as the Strong Brumer-Stark Conjecture turns out to be false in general. Indeed, in [P5] we construct a class of abelian extensions $K / k$ of characteristic $p$ function fields and sets $S_{0}$, such that $\left(K / k, S_{0}\right)$ satisfies hypotheses $\left(\mathrm{H}_{0}\right) 1$ and

$$
\operatorname{Ann}_{\mathbb{Z}[G]}\left(\mu_{K}\right) \cdot \Theta_{S_{0}}(0) \not \subset \operatorname{Fit}_{\mathbb{Z}_{(p)}[G]}\left(A_{S_{0}} \otimes \mathbb{Z}_{(p)}\right)
$$


(see $[\mathbf{P 5}]$ ). Therefore, Lemma 1.2.2 implies that there exists a set $T$ such that $\left(K / k, S_{0}, T\right)$ satisfies hypotheses $\left(\mathrm{H}_{0}\right)$ and

$$
\Theta_{S_{0}, T}(0)=\delta_{T} \cdot \Theta_{S_{0}}(0) \notin \mathrm{Fit}_{\mathbb{Z}_{(p)}[G]}\left(A_{S_{0}} \otimes \mathbb{Z}_{(p)}\right) .
$$

On the other hand, the exact sequence (3) shows that we have a surjective morphism of $\mathbb{Z}[G]$-modules

$$
A_{S_{0}, T} \longrightarrow A_{S_{0}} \longrightarrow 0
$$

Consequently, some elementary properties of Fitting ideals (see $[\mathbf{P 2}]$ ) imply that

$$
\mathrm{Fit}_{\mathbb{Z}_{(p)}[G]}\left(A_{S_{0}, T} \otimes \mathbb{Z}_{(p)}\right) \subseteq \mathrm{Fit}_{\mathbb{Z}_{(p)}[G]}\left(A_{S_{0}} \otimes \mathbb{Z}_{(p)}\right) .
$$

This inclusion combined with (4) implies that, for the class of examples constructed in $[\mathbf{P 5}], \mathbb{Z}_{(p)} \operatorname{SBr} S t\left(K / k, S_{0}\right)$ is false. However, as the reader will see below, conjecture $\mathbb{Z}_{(p)} B(K / k, S, T, r)$ was proved by other means in this case.

In the following sections we will present evidence in support of Rubin's Conjecture, dividing the cases where the conjecture is now proved into two main classes: characteristic $p$ global fields (i.e. function fields over characteristic $p$ finite fields) and characteristic 0 global fields (i.e. number fields).

3.1. Characteristic $p$ global fields. In what follows, $q$ will denote a fixed natural power of a fixed prime number $p, \mathbb{F}_{q}$ denotes the finite field with $q$ elements, and $\mathbb{F}$ is an algebraic closure of $\mathbb{F}_{q}$. As in the previous sections, $(K / k, S, T, r)$ will denote a set of data satisfying hypotheses $\left(H_{r}\right)$, the only difference being that throughout the current section $k$ will be a function field whose exact field of constants is $\mathbb{F}_{q}$ (i.e. $k$ is a finite extension of a field $\mathbb{F}_{q}(T)$, where $T$ is a variable, and $\mathbb{F}_{q}$ is algebraically closed in $k$.) In this context, the $G$-equivariant $L$-function $\Theta_{S, T}(s)$ has a very useful geometric interpretation in terms of $\ell$-adic étale homology of $1-$ motives, due to Deligne and based on Weil's conjectures (proved by Weil himself in this context). In what follows, we briefly describe Deligne's constructions (see $[\mathbf{T a} 4]$ and $[\mathbf{P 1 0}]$ for more details).

To the Galois extension $K / k$ one can associate a canonical Galois cover

$$
X_{1} \stackrel{\pi}{\longrightarrow} Y_{1}
$$

of 1-dimensional smooth projective schemes over $\operatorname{Spec}\left(\mathbb{F}_{q}\right)$ (i.e. smooth projective curves defined over $\left.\mathbb{F}_{q}\right)$, such that their fields of $\mathbb{F}_{q}$-rational functions are $\mathbb{F}_{q}\left(X_{1}\right)=$ $K$ and $\mathbb{F}_{q}\left(Y_{1}\right)=k$ and such that the algebraic morphism of $\operatorname{Spec}\left(\mathbb{F}_{q}\right)$-schemes $\pi$ induces the inclusion $k \subseteq K$ at the level of fields of $\mathbb{F}_{q}$-rational functions. By extending scalars to the algebraic closure $\mathbb{F}$, one obtains a Galois cover of smooth projective schemes of dimension 1 over $\mathbb{F}$

$$
X_{1} \times_{\mathbb{F}_{q}} \mathbb{F}=: X \stackrel{\pi \times_{\mathbb{F}} 1_{\mathbb{F}}}{\longrightarrow} Y:=Y_{1} \times_{\mathbb{F}_{q}} \mathbb{F} .
$$

The reader will notice that, since $\mathbb{F}_{q}$ is algebraically closed in $k$ but not necessarily in $K$, the curve $X$ is always connected whereas $Y_{1}$ will have

$$
\operatorname{card}\left(\operatorname{Spec}\left(K \otimes_{\mathbb{F}_{q}} \mathbb{F}\right)\right)
$$

(mutually isomorphic) connected components. Therefore, the group $\operatorname{Div}(X)$ of divisors on $X$ is a direct sum of the divisor groups of the various connected components of $X$ and $\operatorname{Div}^{0}(X)$ denotes the group of divisors of multidegree 0 (i.e degree 0 on 
each connected component) of $X$. One has a corresponding direct sum splitting of the group of $\mathbb{F}$-rational points

$$
\operatorname{Jac}_{X}(\mathbb{F}):=\operatorname{Div}^{0}(X) / \mathrm{P}(X)
$$

of the Jacobian variety of $X$, where $\mathrm{P}(X)$ denotes the group of principal divisors on $X$. We will denote by $X_{T}$ and $X_{S}$ the (finite) sets of points on $X$ sitting above points on $Y_{1}$ corresponding to primes in $T$ and $S$ respectively. The scheme $X$ is endowed with a natural $G$-action (inherited from $X_{1}$, by extension of scalars) and an action of the $q$-power geometric Frobenius morphism, denoted in what follows by $F_{X}$. The actions of $F_{X}$ and $G$ on $X$ commute and induce commuting linear actions on the various $\ell$-adic etale homology (for $\ell$ prime, $\ell \neq p$ ) and $p$-adic crystalline cohomology groups

$$
\mathrm{H}_{i}\left(X, \mathbb{Z}_{\ell}\right), \quad \mathrm{H}_{\text {cris }}^{i}\left(X / W_{p}\right), \quad i=0,1,2,
$$

associated to $X$, where $\mathbb{Z}_{\ell}$ is the ring of $\ell$-adic integers, $\mathbb{Q}_{\ell}$ its field of fractions, and $W_{p}$ is the $p$-adic ring of Witt vectors. To $X$ and $X_{T}$, Deligne associates a semiabelian variety (i.e. an extension of an abelian variety by a torus) $J_{X, T}$, whose group of $\mathbb{F}$-rational points can be explicitly described as follows

$$
J_{X, T}(\mathbb{F}):=\operatorname{Div}^{0}\left(X \backslash X_{T}\right) /\left\{\operatorname{div}(f) \mid f \in \mathbb{F}(X)^{\times}, f(w)=1, \forall w \in X_{T}\right\} .
$$

We have an obvious exact sequence of $\mathbb{Z}[G]\left[F_{X}\right]$-modules

$$
0 \longrightarrow \Gamma\left(X_{T}, O_{X_{T}}^{\times}\right) / \Gamma\left(X, O_{X}^{\times}\right) \longrightarrow J_{X, T}(\mathbb{F}) \longrightarrow J_{J_{X}}(\mathbb{F}) \longrightarrow 0,
$$

where $O_{X}^{\times}$is the sheaf of invertible regular functions on $X, \Gamma\left(X, O_{X}^{\times}\right)$its group of global sections, viewed inside the group of invertible rational functions $\Gamma\left(X_{T}, O_{X_{T}}^{\times}\right)$ of the closed subscheme $X_{T}$ via restriction. The leftmost non-trivial map is the usual divisor-class map. The exact sequence above makes the semi-abelian variety structure of $J_{X, T}$ explicit (as an extension of the abelian variety $\mathrm{Jac}_{X}$ fully determined by $X$, and the torus on the left, determined by $X$ and $X_{T}$.)

To the set $X_{S}$ we can associate the free $\mathbb{Z}$-module of finite rank $L_{S}$ of divisors of multidegree 0 on $X$, supported at points in $X_{S}$. In other words,

$$
L_{S}:=\operatorname{Div}^{0}\left(X_{S}\right) .
$$

We are now ready to define Deligne's $1-$ motive $M_{K / k, S, T}$ associated to $(K / k, S, T)$.

Definition 3.1.1. The 1 -motive $M_{K / k, S, T}$ consists of the semiabelian variety $J_{X, T}$, the free $\mathbb{Z}$-module $L_{S}$ and the group morphism

$$
L_{S} \stackrel{\widehat{\operatorname{div}}}{\longrightarrow} J_{X, T}(\mathbb{F}),
$$

given by the usual divisor-class map.

To $M_{K / k, S, T}$ and every prime number $\ell \neq p$, one can associate the $\ell$-adic Tate-module $T_{\ell}\left(M_{K / k, S, T}\right)$ in the usual manner (see [Ta4] or [P10]). This comes endowed with natural commuting $G$ and $F_{X}$-actions and fits into a short exact sequence of $\mathbb{Z}_{\ell}[G]\left[F_{X}\right]$-modules

$$
0 \longrightarrow T_{\ell}\left(J_{X, T}\right) \longrightarrow T_{\ell}\left(M_{K / k, S, T}\right) \longrightarrow L_{S} \otimes \mathbb{Z}_{\ell} \longrightarrow 0,
$$

where $T_{\ell}\left(J_{X, T}\right)$ is the $\ell$-adic Tate module obtained from the $\ell$-power torsion points of the semiabelian variety $J_{X, T}$ in the usual manner. The link between the 1-motive 
just constructed and our $(S, T)$-modified $G$-equivariant $L$-function is given by the following (see $[\mathbf{T a} 4]$ for a proof).

Theorem 3.1.2 (Deligne, Weil). We have an equality

$$
\Theta_{S, T}(s)=\operatorname{det}_{\mathbb{Q}_{\ell}[G]}\left(1-q^{-s} \cdot F_{X} \mid T_{\ell}\left(M_{K / k, S, T}\right) \otimes_{\mathbb{Z}_{\ell}} \mathbb{Q}_{\ell}\right) .
$$

for all prime numbers $\ell \neq p$ and all complex numbers $s$.

The main ingredient used in the proof is the Lefschetz trace formula for the $\ell$-adic étale homology (or equivalently cohomology) of $X$ and its endomorphism $F_{X}$, essentially proved by Weil in the 1940s. In particular, this theorem implies that, for all $\ell \neq p$, there exists a polynomial $P_{S, T}^{(\ell)}(u) \in \mathbb{Q}_{\ell}[G][u]$ such that

$$
\Theta_{S, T}(s)=P_{S, T}^{(\ell)}\left(q^{-s}\right), \quad \forall s \in \mathbb{C} .
$$

However, due to the fact that for all primes $v$ in $k, \mathrm{~N}(v)=q^{\operatorname{deg}(v)}$, and for any $n \in \mathbb{Z}$ there exists only finitely many primes $v$ in $k$ with $\operatorname{deg}(v) \leq n, \Theta_{S, T}(s)$ can be written as a convergent infinite product of formal power series in $\mathbb{Z}[G]\left[\left[q^{-s}\right]\right]$ as follows.

$$
\Theta_{S, T}(s)=\prod_{v \in T}\left(1-\sigma_{v}^{-1} \cdot q^{\operatorname{deg}(v)} \cdot\left(q^{-s}\right)^{\operatorname{deg}(v)}\right) \cdot \prod_{v \notin S}\left(1-\sigma_{v}^{-1} \cdot\left(q^{-s}\right)^{\operatorname{deg}(v)}\right)^{-1} .
$$

Equalities (5) and (6) lead to the conclusion that there exists a polynomial $P_{S, T}(u)$ in $\mathbb{Z}[G][u]$, such that $P_{S, T}=P_{S, T}^{(\ell)}$, for all $\ell \neq p$, and

$$
\Theta_{S, T}(s)=P_{S, T}\left(q^{-s}\right), \quad \forall s \in \mathbb{C} .
$$

In particular, this concludes the proof of Theorem 1.2.1 for function fields, as indicated in $\S 1.2$. However, Deligne's theorem implies in fact a much stronger result, namely the Brumer-Stark Conjecture $\operatorname{Br} S t(K / k, S, T)$ in this context. Indeed, (5) implies that, if we set $d:=\operatorname{deg}_{u}\left(P_{S, T}(u)\right)$ and

$$
Q_{S, T}(u):=u^{d} \cdot P_{S, T}\left(u^{-1}\right),
$$

then $Q_{S, T}\left(F_{X}\right)$, viewed as an element in the ring of endomorphisms $\operatorname{End}\left(M_{K / k, S, T}\right)$, annihilates the $\ell$-adic Tate modules $T_{\ell}\left(M_{K / k, S, T}\right)$, for all $\ell \neq p$. This implies right away that, in fact, $Q_{S, T}\left(F_{X}\right)$ annihilates the 1 -motive $M_{K / k, S, T}$ itself, meaning that it annihilates both the group $J_{X, T}(\mathbb{F})$ of $\mathbb{F}$-rational points of the semiabelian variety $J_{X, T}$ and the lattice $L_{S}$. We can now proceed to proving $\operatorname{Br} S t(K / k, S, T)$. For simplicity we will sketch the proof only in the case $\operatorname{card}(S)>1$. The reader can consult [Ta4] for the remaining case. Let $D \in \operatorname{Div}\left(X_{1} \backslash\left(X_{1}\right)_{T}\right)$. We want to show that

$$
\Theta_{S, T}(0) \cdot D=\operatorname{div}\left(\alpha_{D, T}\right)
$$

where $\alpha_{D, T} \in\left(K^{\times}\right)_{0, S_{0}}$, and $\alpha_{D, T} \equiv 1 \bmod { }^{\times} w, \forall w \in T_{K}$. We view $D$ inside $\operatorname{Div}\left(X \backslash X_{T}\right)$ via the natural embedding $\operatorname{Div}\left(X_{1} \backslash\left(X_{1}\right)_{T}\right) \subseteq \operatorname{Div}\left(X \backslash X_{T}\right)$. Since $\operatorname{card}\left(S_{0}\right)>1$, there exist two divisors $\delta$ and $\delta^{\prime}$ with supports which are disjoint and included in $X_{S}$, such that

$$
\operatorname{deg}(D+\delta)=\operatorname{deg}\left(D+\delta^{\prime}\right)=0 .
$$


This implies in particular that $\delta-\delta^{\prime} \in L_{S}$ and therefore $Q_{S, T}\left(F_{X}\right) \cdot\left(\delta-\delta^{\prime}\right)=0$. However, since the supports of $\delta$ and $\delta^{\prime}$ are disjoint, this implies that

$$
Q_{S, T}\left(F_{X}\right) \cdot \delta=Q_{S, T}\left(F_{X}\right) \cdot \delta^{\prime}=0 .
$$

On the other hand, the image of $(D+\delta)$ inside $J_{X, T}(\mathbb{F})$ is annihilated by $Q_{S, T}\left(F_{X}\right)$, which implies that

$$
Q_{S, T}\left(F_{X}\right) \cdot D=\operatorname{div}\left(\alpha_{D, T}\right),
$$

with $\alpha_{D, T} \in \mathbb{F}(X)^{\times}$, and $\alpha_{D, T}(w)=1$, for all $w \in X_{T}$. However, since $D \in$ $\operatorname{Div}\left(X_{1}\right)$, and the points of the scheme $X_{1}$ are fixed by $F_{X}, F_{X}$ acts trivially on $D$. This observation, together with the equality $Q_{S, T}(1)=\Theta_{S, T}(0)$ imply that indeed we can choose $\alpha_{D, T} \in K^{\times}$, such that

$$
\Theta_{S, T}(0) \cdot D=\operatorname{div}\left(\alpha_{D, T}\right),
$$

and $\alpha_{D, T} \equiv 1 \bmod { }^{\times} w, \forall w \in T_{K}$. The condition $\alpha_{D, T} \in\left(K^{\times}\right)_{0, S}$ is automatically satisfied, as the divisor function

$$
\operatorname{div}: \widetilde{K^{\times}} \longrightarrow \operatorname{Div}\left(X_{1}\right)
$$

is an injective morphism of $\mathbb{Z}[G]$-modules and $\operatorname{div}\left(\alpha_{D, T}\right)=\Theta_{S, T}(0) \cdot D$ is in $\operatorname{Div}\left(X_{1}\right)_{0, S}$, because $\Theta_{S, T}(0) \in \mathbb{Z}[G]_{0, S}$ by definition. This concludes our sketch of Deligne's proof of the following.

Theorem 3.1.3 (Deligne, Hayes). If $\operatorname{char}(k)=p>0$, the Brumer-Stark Conjecture $\operatorname{BrSt}\left(K / k, S_{0}\right)$ is true for all $\left(K / k, S_{0}\right)$ satisfying hypothesis $\left(H_{0}\right) 1$.

In $[\mathbf{H 1}]$, Hayes proved the above theorem independently and with methods relying on the theory of rank 1 sign-normalized Drinfeld modules. In fact, Hayes proves a more precise result by explicitly constructing the elements $\alpha_{D}$ whose existence is predicted by Conjecture $\operatorname{Br} S t\left(K / k, S_{0}\right)$ as norms of certain torsion points of a rank 1 sign-normalized Drinfeld module (see [H1] for details.) By combining Theorems 3.1.2 and 2.2.3, we can also conclude the following.

COROllary 3.1.4. Rubin's Conjecture $B(K / k, S, T, r)$ is true if $r=1$ and $\operatorname{char}(k)=p>0$.

In what follows, we will outline the present author's results towards proving Rubin's Conjecture $B(K / k, S, T, r)$ for arbitrary values of $r$. As above, we let $S_{0}=$ $S \backslash\left\{v_{1}, \ldots, v_{r}\right\}$, where $v_{1}, \ldots, v_{r}$ are $r$ distinct primes in $S$ which split completely in $K / k$. In [P8], we refine Deligne's geometric techniques sketched above and prove the following.

Theorem 3.1.5. Let $\ell$ be a prime number, $\ell \neq p$. If for all $i=0,1,2$ the $\ell$-adic étale homology groups $\mathrm{H}_{i}\left(X, \mathbb{Z}_{\ell}\right)$ are $G$-cohomologically trivial, then the $\ell-$ primary part of the Strong Brumer-Stark Conjecture $\mathbb{Z}_{(\ell)} \operatorname{SBrSt}\left(K / k, S_{0}, T\right)$ holds true.

Proposition 3.1 allows us to conclude the following.

TheOREm 3.1.6. Let $\ell$ be a prime number, $\ell \neq p$. If for all $i=0,1,2$ the $\ell$-adic étale homology groups $\mathrm{H}_{i}\left(X, \mathbb{Z}_{\ell}\right)$ are $G$-cohomologically trivial, then the $\ell$-primary part of Rubin's Conjecture $\mathbb{Z}_{(\ell)} B(K / k, S, T, r)$ holds true.

Now, the question to be answered is for what type of extensions $K / k$ the $G^{-}$ cohomological triviality of the $\ell$-adic étale homology of $X$ is satisfied. In order to 
answer this question, we remind the reader that a finite extension $L / F$ of function fields is called a constant field extension if $L$ is obtained by adjoining some roots of unity to $F$ or, equivalently, if $L$ is the compositum $L \cdot \mathbb{F}_{q^{n}}$ of $L$ and some finite extension $\mathbb{F}_{q^{n}}$ of the exact field of constants $\mathbb{F}_{q}$ of $F$.

Definition 3.1.7. Let $\ell$ be a prime number and let $G^{(\ell)}$ be the $\ell$-Sylow subgroup of $G$. The extension $K / k$ is called $\ell$-constant if $K / K^{G^{(\ell)}}$ is a constant field extension. (Here $K^{G^{(\ell)}}$ denotes the maximal subfield of $K$ fixed by $G^{(\ell)}$.)

In $[\mathbf{P 8}]$ we prove the following criterion for cohomological triviality of the $\ell$-adic étale homology of $X$.

Proposition 3.1.8. Let $\ell$ be a prime number. Then $\mathrm{H}_{i}\left(X, \mathbb{Z}_{\ell}\right)$ is $G$-cohom. trivial for all $i=0,1,2$ if and only if the extension $K / k$ is $\ell$-constant.

An immediate consequence of Theorem 3.1.6 and Proposition 3.1.8 is the following result which was also obtained in our earlier paper $[\mathbf{P 2}]$ without any reference to $G$-cohomological triviality.

Corollary 3.1.9. Assume that the set of data $(K / k, S, T, r)$ satisfies hypotheses $\left(H_{r}\right)$. Then

1. Conjecture $\mathbb{Z}[1 /|G|] B(K / k, s, T, r)$ is true.

2. Conjecture $B(K / k, S, T, r)$ is true if $K / k$ is a constant field extension.

Proof (sketch). Indeed, if a prime number $\ell$ is coprime to $|G|$, then $K / k$ is $\ell$-constant, by definition. Also, if $K / k$ is a constant field extension then $K / k$ is $\ell$-constant for all prime numbers $\ell$.

The results obtained by Tan $[\mathbf{T}]$ towards proving generalizations of a conjecture of Gross in characteristic $p$ were used in $[\mathbf{P 8}]$ to prove the following.

TheOREM 3.1.10. Assume that the set of data $(K / k, S, T, r)$ satisfies hypotheses $\left(H_{r}\right)$. Then, conjecture $\mathbb{Z}_{(p)} B(K / k, S, T, r)$ is true.

Theorems 3.1.6, 3.1.10, and Proposition 3.1.8 imply that we can produce examples of extensions $K / k$ where Rubin's Conjecture is true by constructing extensions $K / k$ which are $\ell-$ constant at all primes $\ell \neq p$. For a fixed base field $k$, the compositum of all finite abelian extensions $K / k$ satisfying this property can be obtained by taking the compositum $k_{p \cdot \infty}:=k_{\infty} \cdot k_{p}$ of the maximal abelian pro- $p$ extension $k_{p}$ of $k$ and the maximal constant field extension $k_{\infty}$ of $k$ inside a fixed abelian closure $k^{a b}$ of $k$. Now, based on the definitions, it is easy to show that any finite subextension $K / k$ of $k_{p \cdot \infty} / k$ is $\ell$-constant, for all $\ell \neq p$. Consequently, the following result holds (see $[\mathbf{P} 8]$ ).

THEOREM 3.1.11. Let $k$ be a fixed characteristic $p$ function field and let $k_{p \cdot \infty}$ be the compositum of the maximal constant field extension and the maximal abelian pro-p extension of $k$ inside a fixed abelian closure $k^{a b}$ of $k$. Let $K / k$ be a finite field extension, with $K \subseteq k_{p \cdot \infty}$. Then

1. The $\ell$ part $\mathbb{Z}_{(\ell)} \operatorname{SBr} S t\left(K / k, S_{0}, T\right)$ of the Strong Brumer-Stark Conjecture is true for all $S_{0}$ and $T$ such that $\left(K / k, S_{0}, T\right)$ satisfies hypotheses $\left(H_{0}\right)$.

2. Rubin's Conjecture $B(K / k, S, T, r)$ is true for all $S, T$, and $r$, such that $(K / k, S, T, r)$ satisfies hypotheses $\left(H_{r}\right)$. 
For a fixed base field $k$ and a fixed abelian closure $k^{a b}$, the field $k_{p \cdot \infty}$ sits with a quasi-finite index inside $k^{a b}$, in a sense made precise by the following result whose class-field theoretic proof can be found in $[\mathbf{T}]$ and $[\mathbf{P 8}]$.

Proposition 3.1.12. For any finite, nonempty set $S$ of primes in $k$, let $k_{S}^{a b}$ be the maximal abelian extension of $k$ unramified outside $S$. Then the index

$$
\left[k_{S}^{a b}: k_{S}^{a b} \cap k_{p \cdot \infty}\right]
$$

is finite.

In conclusion, in the case of characteristic $p$ global fields, the $\ell$-primary part $\mathbb{Z}_{(\ell)} B(K / k, S, T, r)$ of Rubin's Conjecture is still unproved precisely in those cases where the conditions

$$
K \not \subset k_{p \cdot \infty}, \quad \ell \| G \mid, \quad \ell \neq p
$$

are simultaneously satisfied. Unfortunately, in theses cases one cannot rely on the link between the $\ell$-primary part of Rubin's Conjecture and the $\ell$-primary part of the Strong-Brumer Stark Conjecture (see Proposition 3.1) due to the fact that the latter statement turns out to be false in these cases in general, as remarked in the introduction to $\S 3$.

REMARK. We would like to conclude this section by noting that, for a fixed base field $k$ and any finite extension $K / k$ such that $K \subseteq k_{p \cdot \infty}$, the group $\mu_{K}$ of roots of unity in $K$ is $G$-cohomologically trivial. This can be easily proved by applying Lemma 2.3.5. If we combine this observation with Corollary 2.3.4(2), we can conclude that, if $K \subseteq k_{p \cdot \infty}$, then

$$
B(K / k, S, T, r) \Longleftrightarrow C(K / k, S, T, r)
$$

for all $(K / k, S, T, r)$ satisfying hypotheses $\left(H_{r}\right)$.

3.2. Characteristic 0 global fields (number fields). In this section we present evidence in support of Rubin's Conjecture $B(K / k, S, T, r)$, in the situation where $\operatorname{char}(k)=0$ (i.e. $k$ is a number field.) We intend to divide the cases for which the conjecture has been proved into two main classes, depending on whether the $r$ primes in $S$ which split completely in $K / k$ are all finite (non-archimedean) or not. Before doing so, we will state a theorem due to Tate and Rubin which escapes this classification.

TheOrem 3.2.1 (Tate, Rubin). Conjecture $B(K / k, S, T, r)$ holds true for any number field $k$ and any extension $K / k$ with $[K: k]=2$.

The theorem above was proved by Tate in the case $r=1$ (see [Ta4]) and extended by Rubin to arbitrary values of $r$ (see [Ru3]). In the case $r=1$, Sands and his collaborators have extended this result to almost arbitrary abelian extensions $K / k$ of Galois group of exponent 2 (see [Sa2] and [DST].) Recently, Sands has also obtained a partial result towards proving conjecture $C(K / k, S, T, r)$ for arbitrary values of $r$ for exponent 2 extensions. We refer the reader to Sands's contribution to this volume for more precise statements of his results.

Now, we return to the classification promised in the introduction.

3.2.1. The $r$ split primes are not all finite. In this case, the following result was proved by Stark in $[\mathbf{S t} \mathbf{I V}]$. 
Theorem 3.2.1.1. Conjecture $B(K / k, S, T, r)$ holds true if $r=1$ and $k$ is either $\mathbb{Q}$ or a quadratic imaginary field $\mathbb{Q}(\sqrt{-d})$, for $d \in \mathbb{Z}_{\geq 1}$.

If $k=\mathbb{Q}, K=\mathbb{Q}\left(\zeta_{m}\right)^{+}$(the maximal real subfield of the full cyclotomic field $\left.\mathbb{Q}\left(\zeta_{m}\right)\right)$, and

$$
S:=\{\ell \mid \ell \text { prime divisor of } m\} \cup\{\infty\},
$$

then the unit $\varepsilon_{S, T}$ is given by

$$
\varepsilon_{S, T}:=\left(\epsilon_{m}^{\delta_{T}}\right)^{1 / 2},
$$

where $\epsilon_{m}$ is the cyclotomic element $\left(1-\zeta_{m}\right)\left(1-\zeta_{m}^{-1}\right)$ and the $1 / 2$ power in the expression above is to be viewed as the unique square root of $\epsilon_{m}^{\delta_{T}}$ in $\mathbb{Q}\left(\zeta_{m}\right)^{+}$which is congruent to 1 modulo every prime in $T_{K}$. For a general real abelian extension $K / \mathbb{Q}$, the units $\epsilon_{S, T}$ is an $(S, T)$-modified version of the norm down to $K$ of the cyclotomic elements given by $(7)$ above, for $m=\mathfrak{f}_{K / \mathbb{Q}}$ (the conductor of $K / \mathbb{Q}$ ).

In the case where $k$ is a quadratic imaginary field and $K$ is a full ray classfield of $k$, Stark shows that the elements $\varepsilon_{S, T}$ are essentially elliptic units. For an arbitrary abelian extension $K / k$, the elements $\varepsilon_{S, T}$ are essentially norms down to $K$ of elliptic units of the minimal ray class-field containing $K$.

The following result is an obvious consequence of Theorems 3.2.1 and 2.3.2.

TheOrem 3.2.1.2. Assume that $(K / k, S, 1)$ satisfies hypotheses $\left(H_{1}\right) 1-3$, and $k$ is either $\mathbb{Q}$ or a quadratic imaginary field. Let $k^{\prime}$ denote an intermediate field $k \subseteq k^{\prime} \subseteq K$, and let $r:=\left[k^{\prime}: k\right]$. Then, conjecture $C\left(K / k^{\prime}, S_{k^{\prime}}, r\right)$ holds true.

Unfortunately, aside from Theorem 3.2.1.2 and its consequences pertaining to Conjecture B under the hypothesis of cohomological triviality for $\mu_{K}$ (see Corollary 2.3.4), under the current hypotheses we have practically no information on Rubin's Conjecture for $r>1$ at present, except for a few partial results which do not deserve to be put into print yet.

A proof of Conjecture $\mathrm{B}$ or $\mathrm{C}$ in cases where two infinite primes in $k$ split completely in $K / k$ and which do not arise via base-change from order of vanishing 1 cases (as described in Theorem 3.2.1.2) would consist a major advance in the subject.

3.2.2. The $r$ split primes are finite. Under the assumption in the title, the base field $k$ is forced to be a totally real number field (i.e. all its embeddings in $\mathbb{C}$ are in fact embeddings in $\mathbb{R}$ ) and $K$ a totally imaginary number field (i.e. $K$ admits no embedding in $\mathbb{R}$.) Consequently, throughout $§ 3.2 .2$ we will assume that $k$ is totally real and $K$ is totally imaginary. A classical result in this case is the following theorem proved by Stark (see [St IV ]), as a direct consequence of the classical Stickelberger Theorem (see $[\mathbf{C o}]$ ).

Theorem 3.2.2.1. Conjecture $B(K / k, S, T, r)$ holds true if $k=\mathbb{Q}, K$ is an imaginary field and $r=1$.

For example, let us assume that $K:=\mathbb{Q}\left(\zeta_{m}\right)$, where $m \in \mathbb{Z}_{>1}$, and

$$
S:=\{\ell \mid \ell \text { prime divisor of } m\} \cup\{\infty\} \cup\{p\},
$$

where $p$ is a prime which splits completely in $\mathbb{Q}\left(\zeta_{m}\right) / \mathbb{Q}$. Let $\wp$ be a prime in $\mathbb{Q}\left(\zeta_{m}\right)$ dividing $p$. Then, the unique element $\varepsilon_{S, T} \in\left(U_{K, S, T}\right)_{1, S}$ determined by this choice of $\wp$ is given by

$$
\varepsilon_{S, T}:=\left(g_{\wp}^{\delta_{T}}\right)^{1 / w_{m}}
$$


where $g_{\wp}$ is a normalized Gauss sum associated to $\wp$ in $\mathbb{Q}\left(\zeta_{m}\right)$ and $w_{m}$ is the number of roots of unity in $\mathbb{Q}\left(\zeta_{m}\right)$. The $1 / w_{m}$ power above is to be viewed as the unique $w_{m}$-root of $g_{\wp}^{\delta_{T}}$ in $\mathbb{Q}\left(\zeta_{m}\right)$ which is congruent to 1 modulo every single prime in $T_{K}$.

If $k$ is an arbitrary totally real number field, then the following two theorems (see $[\mathbf{P} 11]$ and $[\mathbf{P 6}]$ ) provide useful reduction steps in our attempt to prove Rubin's Conjecture under the present assumptions. The first reduction is the following natural generalization to arbitrary values of $r$ of the main result in $[\mathbf{Y}]$ (proved in loc. cit. for $r=1$.)

Theorem 3.2.2.2. Assume that $(K / k, S, T, r)$ satisfies hypotheses $\left(H_{r}\right)$, with $k$ totally real and $K$ totally imaginary number fields. Let $K_{C M}$ be the maximal $C M$-subfield of $K$. Then the following implication holds true.

$$
\mathbb{Z}[1 / 2] B\left(K_{C M} / k, S, T, r\right) \Longleftrightarrow \mathbb{Z}[1 / 2] B(K / k, S, T, r) .
$$

We remind the reader that a CM-field is a totally imaginary field which is a quadratic extension of a totally real field. The decomposition groups of all the infinite primes of a CM-field $L$ over any totally real subfield are equal and generated by the field's unique "complex conjugation" endomorphism, denoted in what follows by $j_{L}$. In particular, under the assumptions and notations in Theorem 3.2.2.2, $K_{C M}$ is the maximal subfield of $K$ fixed by the subgroup $H$ of $G$ generated by all the products $j_{v} \cdot j_{v^{\prime}}$, where $v$ and $v^{\prime}$ are infinite primes in $k$ and $j_{v}$ and $j_{v^{\prime}}$ are the generators of their (order 2) decomposition groups $G_{v}$ and $G_{v^{\prime}}$ in $G:=$ $\operatorname{Gal}(K / k)$. Since in what follows we will be concerned only with "the odd part" $\mathbb{Z}[1 / 2] B(K / k, S, T, r)$ of Rubin's conjecture (with some exceptions which will be pointed out when appropriate), Theorem 3.2.2.2 permits us to make the additional assumption that $K$ is a CM-field. For simplicity (and following $[\mathbf{P 6}]$ ), we let $j:=j_{K}$ (viewed as an element in $G$ ), and

$$
R_{-}:=\mathbb{Z}[G] /(1+j), \quad R^{-}:=(1-j) \mathbb{Z}[G] .
$$

The reader will notice right away that $R_{-}$and $R^{-}$are the largest quotient module and respectively submodule of $\mathbb{Z}[G]$ on which $j$ acts as multiplication by -1 , which justifies the notation. We have an obvious $\mathbb{Z}[G]$-module isomorphism

$$
\frac{1}{2} \pi: R^{-} \stackrel{\sim}{\longrightarrow} R_{-},
$$

sending $(1-j) \cdot x$ to $(x \bmod (1+j))$, for all $x \in \mathbb{Z}[G]$. For every $\mathbb{Z}[G]$-module $M$ and every prime number $\ell$, we let

$$
M_{-}:=M \otimes_{\mathbb{Z}[G]} R_{-}, \quad R_{(\ell),-}:=R_{-} \otimes \mathbb{Z}_{(\ell)} .
$$

Let $\left\{v_{1}, \ldots v_{r}\right\}$ be a set of $r$ distinct primes in $S$ which split completely in $K / k$. Since, under the present assumptions, the $v_{i}$ 's are all finite, if we let $S_{0}:=S \backslash$ $\left\{v_{1}, \ldots, v_{r}\right\}$, then $\left(K / k, S_{0}, T\right.$, ) satisfies hypotheses $\left(H_{0}\right)$. Since $\Theta_{S_{0}, T}(0)$ belongs to $\mathbb{Z}[G]_{0, S_{0}}$ and $\mathbb{Z}[G]_{0, S_{0}} \subseteq R^{-}$(apply Lemma 1.2.3 and keep in mind that $G_{v}=\{1, j\}$, for all infinite $v \in S_{0}$ ), we can conclude that

$$
\Theta_{S_{0}, T}(0) \subseteq R^{-} .
$$

Consequently, the element $\frac{1}{2} \pi\left(\Theta_{S_{0}, T}(0)\right) \in R_{-}$is well defined. In what follows, $S_{\infty}$ denotes the set of infinite primes in $k$ and $A_{K, T}:=A_{S_{\infty}, T}$. The second reduction 
step used in the proof of Rubin's Conjecture in the cases to be described below is given by the following theorem, proved in $[\mathbf{P 6}]$.

TheOREM 3.2.2.3. Let $\ell$ be a prime number. The following hold true.

1. $\frac{1}{2} \pi\left(\Theta_{S_{0}, T}(0)\right) \in \operatorname{Fit}_{R_{(\ell),-}}\left(\left(A_{K, T} \otimes \mathbb{Z}_{(\ell)}\right)_{-}\right) \Longrightarrow \mathbb{Z}_{(\ell)} B(K / k, S, T, r)$.

2. $\frac{1}{2} \pi\left(\Theta_{S_{0}, T}(0)\right) \in \operatorname{Fit}_{R_{-}}\left(\left(A_{K, T}\right)_{-}\right) \Longrightarrow B(K / k, S, T, r)$.

In the above theorem, it is obvious that (2) is a consequence of (1) for all primes $\ell$. Since it implies the Brumer-Stark Conjecture $\operatorname{Br} S t\left(K / k, S_{0}, T\right)$, the left-hand side of the implication in (1) will be called the Strong Brumer-Stark Conjecture over $R_{-}$. We will use the notation

$$
S B r S t_{R_{-}}\left(K / k, S_{0}, T\right): \frac{1}{2} \pi\left(\Theta_{S_{0}, T}(0)\right) \in \operatorname{Fit}_{R_{-}}\left(\left(A_{K, T}\right)_{-}\right) .
$$

As before, we attach the obvious meaning to $\mathbb{Z}_{(\ell)} S B r S t_{R_{-}}\left(K / k, S_{0}, T\right)$, for all prime numbers $\ell$.

Assuming that $k=\mathbb{Q}$, in $[\mathbf{P 9}]$ we use Iwasawa theoretic techniques recently introduced by Kurihara in $[\mathbf{K u}]$ to prove the following.

Theorem 3.2.2.4. Assume that $\ell$ is an odd prime number, $K$ is a $C M$ field, and $\left(K / \mathbb{Q}, S_{0}, T\right)$ satisfies hypotheses $\left(H_{0}\right)$. Then, we have the following.

1. Statement $\mathbb{Z}_{(\ell)} S B r S t_{R_{-}}\left(K / \mathbb{Q}, S_{0} \cup\{\ell\}, T\right)$ holds true.

2. If $\ell \times|G|$, then statement $\mathbb{Z}_{(\ell)} S B r S t_{R_{-}}\left(K / \mathbb{Q}, S_{0}, T\right)$ holds true.

The reader familiar with Iwasawa descent techniques will sense right away the reason why one needs to add the prime $\ell$ to the set $S_{0}$ in Theorem 3.2.2.4(1). Roughly speaking, the approach to proving (1) is as follows: one proves an analogous statement linking the value at $s=0$ of the $T$-modified $G$-equivariant $\ell$-adic $L$ function, to the Fitting ideal over the ring $\Lambda[G]_{-}$of a certain $\Lambda[G]_{-}-$module, where $\Lambda$ is the 1 -variable Iwasawa algebra associated to the cyclotomic $\mathbb{Z}_{\ell}-$ extension of $K$. Then, one projects (descends) this statement onto the 0 -level of the Iwasawa tower, obtaining this way a statement about the value at $s=0$ of the global $G$-equivariant $L$-function. However, since in the $\ell$-adic interpolation construction leading to the $G$-equivariant $\ell$-adic $L$-function one is forced to interpolate values of global $L$ functions with Euler factors at primes in $S_{0} \cup\{\ell\}$ removed, the descent leads to $\Theta_{S_{0} \cup\{\ell\}, T}(0)$ rather than $\Theta_{S_{0}, T}(0)$. The following is an immediate consequence of Theorems 3.2.2.3 and 3.2.2.4.

Corollary 3.2.2.5. Assume that $K$ is a $C M$ field and $(K / \mathbb{Q}, S, T, r)$ satisfies hypotheses $\left(H_{r}\right)$. Then

1. $\mathbb{Z}[1 /|G|] B(K / \mathbb{Q}, S, T, r)$ holds true.

2. $\mathbb{Z}[1 / 2] B(K / \mathbb{Q}, S, T, r)$ holds true for all $S$ containing all odd primes $\ell$, such that $\ell|| G \mid$.

In order to complete the proof of Rubin's Conjecture for abelian CM extensions $K / \mathbb{Q}$, one needs to solve two major problems: firstly, eliminate the $\ell$-imprimitivity phenomenon in Theorem 3.2.2.4(1), for all odd primes $\ell$ dividing $|G|$, and secondly attack the 2 -primary part " $\mathbb{Z}_{(2)} B$ " of the conjecture. These problems are very difficult and have not been solved in full generality yet. However, an application of the so called "two variable $\ell$-adic $L$-function" techniques developed by Wiles in [Wi2] and perfected by Greither in [Gre2] has enabled us to solve the $\ell$-imprimitivity 
problem under some extra hypotheses, as described in the result below (see [P9] for a proof).

THEOREM 3.2.2.6. Let $K$ be a $C M$-abelian extension of $\mathbb{Q}$ of Galois group $G$. Assume that at least one of the following conditions is satisfied.

(a) For all odd primes $\ell$, such that $\ell|| G \mid$, one has $j_{K} \in G_{\ell}$.

(b) For all odd primes $\ell$ which ramify in $K / \mathbb{Q}$, one has $j_{K} \in G_{\ell}$.

Then, we have the following.

1. Conjecture $\mathbb{Z}_{(\ell)} S B r S t_{R_{(\ell),-}}\left(K / \mathbb{Q}, S_{0}, T\right)$ holds true whenever $\left(K / \mathbb{Q}, S_{0}, T\right)$ satisfies hypotheses $\left(H_{0}\right)$.

2. Conjecture $\mathbb{Z}[1 / 2] B(K / \mathbb{Q}, S, T, r)$ holds true whenever $(K / \mathbb{Q}, S, T, r)$ satisfies hypotheses $\left(H_{r}\right)$.

The reader will notice right away that extensions $K / \mathbb{Q}$ with $K \subseteq \mathbb{Q}\left(\mu_{p^{n}}\right)$ with $p \neq 2$ prime satisfy condition (b) in the theorem above. For this type of extensions, results of Greither [Gre1] have lead us in $[\mathbf{P 7}]$ to a solution of the 2-primary part problem stated above as well.

TheOREm 3.2.2.7. Let $p$ be an odd prime and $K \subseteq \mathbb{Q}\left(\mu_{p^{n}}\right)$ a $C M$-field. Then

1. $S B r S t_{R_{-}}\left(K / \mathbb{Q}, S_{0}, T\right)$ holds true if $\left(K / \mathbb{Q}, S_{0}, T\right)$ satisfies hypotheses $\left(H_{0}\right)$.

2. $B(K / \mathbb{Q}, S, T, r)$ holds true if $(K / \mathbb{Q}, S, T, r)$ satisfies hypotheses $\left(H_{r}\right)$.

Now, we move on to the case where the base field $k$ is a fixed general totally real number field. In his work on Brumer's Conjecture [Gre2], Greither introduced a special class of CM extensions of such fields $k$, called "nice extensions". We define this class below.

Definition 3.2.2.8. Let $K$ be a CM-field, such that $K / k$ is an abelian extension of Galois group $G$. Let $K^{c l}$ be the Galois closure over $\mathbb{Q}$ of $K$. A finite prime $\wp$ in $k$ of odd residual characteristic $p$ is called critical, if it satisfies at least one of the following conditions.

(a) $K^{c l} \subseteq K^{c l,+}\left(\mu_{p}\right)$, where $K^{c l,+}$ is the maximal totally real subfield of $K^{c l}$.

(b) $\wp$ ramifies in $K / k$.

Definition 3.2.2.9. An abelian extension $K / k$ of Galois group $G$, with $K \mathrm{CM}$ and $k$ totally real is called a nice extension if it satisfies the following conditions.

1. $j_{K} \in G_{\wp}$, for all critical primes $\wp$ of $k$.

2. $\mu_{K} \otimes \mathbb{Z}[1 / 2]$ is $G$-cohomologically trivial.

REMARK. By applying Lemma 2.3.5, the reader will notice that one can produce examples of nice extensions, for example, as follows (see [P6]).

If $k$ is a totally real field and $\ell$ a prime number which is unramified in $k / \mathbb{Q}$, then $k\left(\mu_{\ell^{n}}\right) / k$ is nice, for all $n$.

Let $k$ be a Galois extension of $\mathbb{Q}$ of odd degree. Let $\mathfrak{a}$ be an ideal in $O_{k}$, stable under the action of $\operatorname{Gal}(k / \mathbb{Q})$. Let $\operatorname{Supp}(\mathfrak{a})$ denote the set of residual characteristics of all the primes dividing $\mathfrak{a}$. Let $k(\mathfrak{a})$ denote the maximal abelian extension of $k$, of conductor dividing $\mathfrak{a}$, and of degree coprime to $2 \prod^{\prime} p$, where the product is taken over all primes $p$ in $\operatorname{Supp}(\mathfrak{a})$. Then, $k(\mathfrak{a})\left(\sqrt{-\prod^{\prime} p}\right) / k$ is a nice extension.

Throughout the remainder of this section, we make the following assumption: For all odd primes $\ell$, the cyclotomic Iwasawa invariant $\mu_{K, \ell}$ of $K$ is equal to 0 . 
The vanishing of the cyclotomic Iwasawa invariants $\mu_{K, \ell}$ is one of the central conjectures in Iwasawa theory. In the case where $K$ is an abelian extension of $\mathbb{Q}$, this conjecture was proved by Ferrero and Washington in $[\mathbf{F W}]$. Very recently, Barsky has announced a proof of this conjecture for all CM fields $K$ (see $[\mathbf{B a 2}]$ ). Under the assumption above, Greither proves in [Gre2] the following.

TheOREM 3.2.2.10 (GREITHER). If $K / k$ is a nice extension and $\left(K / k, S_{0}\right)$ satisfies $\left(H_{0}\right)$, then the "odd part" of Brumer's Conjecture $\mathbb{Z}[1 / 2] \operatorname{Br}\left(K / k, S_{0}\right)$ holds true.

In $[\mathbf{P 6}]$, we use Greither's results and techniques to prove the following.

THEOREM 3.2.2.11. If $K / k$ is a nice extension, then

1. $\mathbb{Z}[1 / 2] S B r S t_{R_{-}}\left(K / k, S_{0}, T\right)$ holds true if $\left(K / k, S_{0}, T\right)$ satisfies $\left(H_{0}\right)$.

2. $\mathbb{Z}[1 / 2] B(K / k, S, T, r)$ holds true if $(K / k, S, T, r)$ satisfies $\left(H_{r}\right)$.

\section{Applications of Rubin's Conjecture}

In this section we will give applications of Rubin's Conjecture to the theory of Euler Systems (following [Ru2], [Ru3] and [Ru4]) and the theory of special units and Gras-type conjectures (following $[\mathbf{R u 3}]$ and $[\mathbf{P 3}]$ ) in quite general contexts.

4.1. Euler Systems. In order to simplify matters, in what follows, $k$ will denote a fixed totally real number field or characteristic $p$ function field. Let $T$ be a fixed, finite, nonempty set of non-archimedean (finite) primes in $k$, containing at least two primes of different residual characteristics, if $\operatorname{char}(k)=0$. We let $S_{\infty}$ denote the set of all archimedean (infinite) primes in $k$, if $\operatorname{char}(k)=0$, and a fixed, finite, non-empty set of primes in $k$, with $T \cap S_{\infty}=\emptyset$, if $\operatorname{char}(k)=p$. We fix once and for all $k, S_{\infty}$, and $T$ as above, and fix a separable closure $k^{\text {sep }}$ of $k$.

Definition 4.1.1. Let $\mathcal{K}$ be the set of all fields $K$, with $k \subseteq K \subseteq k^{s e p}$, such that $K / k$ is a finite abelian extension unramified at primes in $T$, totally split at primes in $S_{\infty}$, and of nontrivial conductor $\mathfrak{f}_{K / k}$.

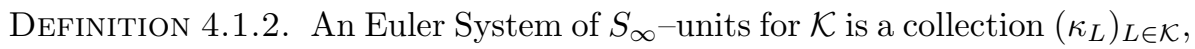
with $\kappa_{L} \in U_{L, S_{\infty}}$, such that, for all $L^{\prime} \subseteq L$ in $\mathcal{K}$, we have

$$
\operatorname{Norm}_{L / L^{\prime}}\left(\kappa_{L}\right)=\left(\prod_{v \mid \mathfrak{f}_{L / k}, v \chi_{\mathfrak{f}_{L^{\prime}} / k}}\left(1-\sigma_{v}^{-1}\right)\right) \cdot \kappa_{L^{\prime}},
$$

where $\sigma_{v}$ is the Frobenius morphism associated to $v$ in $G\left(L^{\prime} / k\right)$.

In this section, we follow $[\mathbf{R u 3}]$ to show how one can construct non-trivial Euler Systems of $S_{\infty}$-units for $\mathcal{K}$, assuming that Rubin's Conjecture holds true. For every $K \in \mathcal{K}$, let $S_{K}:=S_{\infty} \cup\left\{\mathfrak{p} \mid \mathfrak{p}\right.$ prime in $\left.k, \mathfrak{p} \mid \mathfrak{f}_{K / k}\right\}$. We also let $r:=\operatorname{card}\left(S_{\infty}\right)$. Then, the choices we have made force the sets of data $\left(K / k, S_{K}, T, r\right)$, for all $K \in \mathcal{K}$, to satisfy hypotheses $\left(H_{r}\right)$. Throughout this section, we work under the assumption that Rubin's Conjecture $B\left(K / k, S_{K}, T, r\right)$ is true, for all $K \in \mathcal{K}$. For every $K \in \mathcal{K}$, we let $\varepsilon_{K}:=\varepsilon_{K / k, S_{K}, T}$ be the (unique) element in Rubin's lattice $\Lambda_{K}$ associated to $\left(K / k, S_{K}, T, r\right)$, verifying conjecture $B\left(K / k, S_{K}, T, r\right)$. Also, let $U_{K}:=U_{K, S, T}$ and

$$
U_{K}^{*}:=\operatorname{Hom}_{\mathbb{Z}[G(K / k)]}\left(U_{K}, \mathbb{Z}[G(K / k)]\right),
$$

for all $K \in \mathcal{K}$. 
Let $K, K^{\prime} \in \mathcal{K}$, such that $K^{\prime} \subseteq K$. Then, we have norm maps

$$
U_{K} \stackrel{N_{K / K^{\prime}}}{\longrightarrow} U_{K^{\prime}}, \quad U_{K}^{*} \stackrel{N_{K / K^{\prime}}^{*}}{\longrightarrow} U_{K^{\prime}}^{*}
$$

where $N_{K / K^{\prime}}$ is the usual norm from $K^{\times}$down to $\left(K^{\prime}\right)^{\times}$restricted to $U_{K}$, and $N_{K / K^{\prime}}^{*}$ is defined as follows. For every $\phi \in U_{K}^{*}$, we let

$$
N_{K / K^{\prime}}^{*}(\phi):=\frac{1}{\left[K: K^{\prime}\right]} \cdot \pi_{K / K^{\prime}} \circ \phi \circ i_{K / K^{\prime}},
$$

where $\pi_{K / K^{\prime}}: \mathbb{Z}[G(K / k)] \longrightarrow \mathbb{Z}\left[G\left(K^{\prime} / k\right)\right]$ is the canonical projection map induced by restriction at the level of Galois groups and $i_{K / K^{\prime}}: U_{K^{\prime}} \longrightarrow U_{K}$ is the inclusion map. The factor $1 /\left[K: K^{\prime}\right]$ in the above definition is justified by the fact that for all $\phi \in U_{K}^{*}$ and all $u \in U_{K^{\prime}}$, one has

$$
\phi(u) \in \mathbb{Z}[G(K / k)]^{G\left(K / K^{\prime}\right)}=N_{G\left(K / K^{\prime}\right)} \cdot \mathbb{Z}[G(K / k)],
$$

where $N_{G\left(K / K^{\prime}\right)}:=\sum_{\sigma \in G\left(K / K^{\prime}\right)} \sigma$. Consequently,

$$
\pi_{K / K^{\prime}} \circ \phi \circ i_{K / K^{\prime}}(u) \in\left[K: K^{\prime}\right] \cdot \mathbb{Z}\left[G\left(K^{\prime} / k\right)\right]
$$

therefore

$$
\frac{1}{\left[K: K^{\prime}\right]} \cdot \pi_{K / K^{\prime}} \circ \phi \circ i_{K / K^{\prime}}(u) \in \mathbb{Z}\left[G\left(K^{\prime} / k\right)\right] .
$$

However, a more conceptual justification of the definition of $N_{K / K^{\prime}}^{*}$ can be given as follows. Since $U_{K}$ and $U_{K^{\prime}}$ have no $\mathbb{Z}$-torsion (a consequence of properties satisfied by the set $T$ ), we have canonical abelian group isomorphisms

$$
\operatorname{Hom}_{\mathbb{Z}}\left(U_{K}, \mathbb{Z}\right) \stackrel{\sim}{\longrightarrow} U_{K}^{*}, \quad \operatorname{Hom}_{\mathbb{Z}}\left(U_{K^{\prime}}, \mathbb{Z}\right) \stackrel{\sim}{\longrightarrow} U_{K^{\prime}}^{*}
$$

which associate to every $\psi \in \operatorname{Hom}_{\mathbb{Z}}\left(U_{K}, \mathbb{Z}\right)$ the element $\widehat{\psi} \in U_{K}^{*}$, defined by

$$
\widehat{\psi}(u):=\sum_{\sigma \in G(K / k)} \psi\left(u^{\sigma^{-1}}\right) \cdot \sigma
$$

for all $u \in U_{K}$, and similarly at the $K^{\prime}$-level. As the reader can easily check, $N_{K / K^{\prime}}^{*}$ defined above is the unique map which makes the diagram

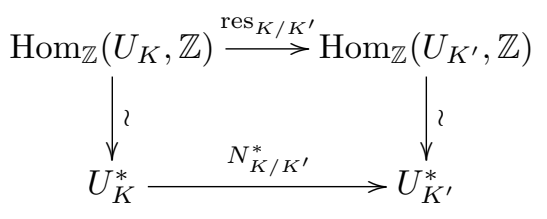

commutative, where $\operatorname{res}_{K / K^{\prime}}$ is the usual restriction of a function defined on $U_{K}$ to its subgroup $U_{K^{\prime}}$. Interpreting $N_{K / K^{\prime}}^{*}$ in terms of the commutative diagram above proves to be beneficial from yet another point of view, made explicit below.

LEMMA 4.1.3. Under the assumptions and notations introduced above, the maps

$$
N_{K / K^{\prime}}^{*}: U_{K}^{*} \longrightarrow U_{K^{\prime}}^{*}
$$

are surjective, for all $K, K^{\prime} \in \mathcal{K}$. 
Proof. It follows at once from Galois theory and the way $T$ was chosen that the quotient group $U_{K} / U_{K^{\prime}}$ has no $\mathbb{Z}$-torsion. This implies that the restriction map $\operatorname{res}_{K / K^{\prime}}$ is surjective. Since the diagram above is commutative, $N_{K / K^{\prime}}^{*}$ is surjective as well.

We let $\left(N_{K / K^{\prime}}^{*}\right)^{(r-1)}:=\left(N_{K / K^{\prime}}^{*}, \ldots, N_{K / K^{\prime}}^{*}\right)$, viewed as (surjective) norm maps

$$
\left(N_{K / K^{\prime}}^{*}\right)^{(r-1)}:\left(U_{K}^{*}\right)^{(r-1)} \longrightarrow\left(U_{K^{\prime}}^{*}\right)^{(r-1)},
$$

at the level of direct sums of duals of groups of units. The following is proved in [Ru3].

Proposition 4.1.4. For all $\Phi=\left(\Phi_{L}\right)_{L \in \mathcal{K}} \in \underset{L \in \mathcal{K}}{\varliminf_{L}}\left(U_{L}^{*}\right)^{(r-1)}$, and all $K, K^{\prime} \in \mathcal{K}$, such that $K^{\prime} \subseteq K$, one has the following equality in $U_{K^{\prime}}$

$$
N_{K / K^{\prime}}\left(\widetilde{\Phi}_{K}\left(\varepsilon_{K}\right)\right)=\left(\prod_{v \in S_{K} \backslash S_{K^{\prime}}}\left(1-\sigma_{v}^{-1}\right)\right) \cdot \widetilde{\Phi}_{K^{\prime}}\left(\varepsilon_{K^{\prime}}\right),
$$

where the projective limit is taken with respect to the norm maps $\left(N_{. / .}^{*}\right)^{(r-1)}$ and $\sigma_{v}$ denotes the Frobenius morphism of $v \in S_{K} \backslash S_{K^{\prime}}$ inside $G\left(K^{\prime} / k\right)$.

Proof (sketch). This is a direct consequence of the uniqueness of $\varepsilon_{K}$, for all $K \in \mathcal{K}$, and the following functoriality property of $G$-equivariant $L$-functions.

$$
\begin{aligned}
\pi_{K / K^{\prime}}\left(\Theta_{K / k, S_{K}, T}(s)\right) & =\Theta_{K^{\prime} / k, S_{K}, T}(s) \\
& =\left(\prod_{v \in S_{K} \backslash S_{K^{\prime}}}\left(1-\sigma_{v}^{-1} \cdot \mathrm{N} v^{-s}\right)\right) \cdot \Theta_{K^{\prime} / k, S_{K^{\prime}}, T}(s),
\end{aligned}
$$

for all $s \in \mathbb{C}$, where $\pi_{K / K^{\prime}}: \mathbb{C}[G(K / k)] \longrightarrow \mathbb{C}\left[G\left(K^{\prime} / k\right)\right]$ is the canonical $\mathbb{C}$-linear projection induced by the restriction map at the level of Galois groups.

For every $K \in \mathcal{K}$, let $I(G(K / k))$ be the augmentation ideal in the integral group ring $\mathbb{Z}[G(K / k)]$. The projections $\pi_{K / K^{\prime}}$ induce surjective maps

$$
\pi_{K / K^{\prime}}: I(G(K / k)) \longrightarrow I\left(G\left(K^{\prime} / k\right)\right)
$$

at the level of augmentation ideals, for all $K^{\prime} \subseteq K$ in $\mathcal{K}$.

Proposition 4.1.5. The following hold true.

1. For all $K \in \mathcal{K}, \eta_{K} \in I(G(K / k))$, and $\Phi_{K} \in\left(U_{K}^{*}\right)^{(r-1)}$, we have

$$
\eta_{K} \cdot \widetilde{\Phi}_{K}\left(\varepsilon_{K}\right) \in U_{K, S_{\infty}, T}
$$

2. Let $\eta=\left(\eta_{L}\right)_{L \in \mathcal{K}} \in \varliminf_{L \in \mathcal{K}}^{\varliminf_{L}} I(G(L / k))$ and $\Phi=\left(\Phi_{L}\right)_{L \in \mathcal{K}} \in \varliminf_{L \in \mathcal{K}}^{\lim _{L}}\left(U_{L}^{*}\right)^{(r-1)}$, and let $K^{\prime} \subseteq K$ in $\mathcal{K}$. Then

$$
N_{K / K^{\prime}}\left(\eta_{K} \cdot \widetilde{\Phi}_{K}\left(\varepsilon_{K}\right)\right)=\left(\prod_{v \in S_{K} \backslash S_{K^{\prime}}}\left(1-\sigma_{v}^{-1}\right)\right) \cdot\left(\eta_{K^{\prime}} \cdot \widetilde{\Phi}_{K^{\prime}}\left(\varepsilon_{K^{\prime}}\right)\right),
$$

viewed as an equality in $U_{K^{\prime}, S_{\infty}, T}$. 
Proof (sketch). Statement (2) is a direct consequence of Proposition 4.1.4. Statement (1) follows from the fact that for all $K \in \mathcal{K}$ and all $\Phi_{K}$, one has

$$
\Phi_{K}\left(\varepsilon_{K}\right) \in\left(U_{K}\right)_{r, S_{K}}
$$

combined with the observation that

$$
I(G(K / k)) \cdot\left(U_{K}\right)_{r, S_{K}} \subseteq U_{K, S_{\infty}, T} .
$$

Definition 4.1.2 and Proposition 4.1.5 lead us to the following.

Corollary 4.1.6. If Rubin's Conjecture $B\left(K / k, S_{K}, T, r\right)$ holds true for all $K \in \mathcal{K}$, then for all $\eta=\left(\eta_{L}\right)_{L \in \mathcal{K}}$ and $\Phi=\left(\Phi_{L}\right)_{L \in \mathcal{K}}$ as in Proposition 4.1.5(2),

$$
\left(\eta_{L} \cdot \widetilde{\Phi}_{L}\left(\varepsilon_{L}\right)\right)_{L \in \mathcal{K}}
$$

is an Euler System of $S_{\infty}$-units for $\mathcal{K}$.

ExAmPles AND REMARKS. In the case $k=\mathbb{Q}$, the general construction outlined above leads to a slightly modified version of the classical Euler Systems of cyclotomic units (see [Ru1] and [Ru2]). Indeed, in this case $S_{\infty}$ consists of the unique archimedean prime in $\mathbb{Q}$, so $r=1$ and therefore the maps $\widetilde{\Phi}_{K}$ are not present. For a given $T$ as above, the class $\mathcal{K}$ is dominated by $\left(\mathbb{Q}\left(\zeta_{m}\right)^{+}\right)_{m}$ with $m \in \mathbb{Z}_{\geq 3}, m$ coprime to $T$. Therefore, for each $K \in \mathcal{K}, K \subseteq \mathbb{Q}\left(\zeta_{m}\right)^{+}$for some $m$, for example $m=\mathfrak{f}_{K / \mathbb{Q}}$. Proposition 4.1 .4 and comments after the statement of Theorem 3.2.1.1 imply that, if we set $m=\mathfrak{f}_{K / \mathbb{Q}}$, then

$$
\varepsilon_{K}=N_{\mathbb{Q}\left(\zeta_{m}\right)+/ K}\left(\left(\delta_{T, m} \cdot \epsilon_{m}\right)^{1 / 2}\right),
$$

where $\epsilon_{m}$ denotes the cyclotomic element $\left(1-\zeta_{m}\right)\left(1-\zeta_{m}^{-1}\right)$ in $\mathbb{Q}\left(\zeta_{m}\right)^{+}$and $\delta_{T, m}=$ $\prod_{v \in T}\left(1-\sigma_{v}^{-1} \cdot N v\right)$ in $\mathbb{Z}\left[G\left(\mathbb{Q}\left(\zeta_{m}\right)^{+} / \mathbb{Q}\right)\right]$. Therefore, for any $\eta=\left(\eta_{K}\right)_{K \in \mathcal{K}}$ as above, the Euler System given by Proposition 4.1.5 in this case is uniquely determined via norm maps by the norm-coherent cyclotomic elements

$$
\eta_{\mathbb{Q}\left(\zeta_{m}\right)^{+}} \cdot\left(\delta_{T, m} \cdot \epsilon_{m}\right)^{1 / 2}, \quad m \in \mathbb{Z}_{\geq 3}, \quad m \text { coprime to } T .
$$

Of course, we could have performed the same type of constructions, under the assumption that $k$ is a totally imaginary field, the class $\mathcal{K}$ consists of arbitrary abelian extensions $K$ of $k$ of non-trivial conductor and Rubin's Conjecture $B\left(K / k, S_{K}, T, r\right)$ is true for all $K \in \mathcal{K}$. If restricted to the case where $k$ is a quadratic imaginary field, one arrives this way at slightly modified versions of the classical Euler Systems of elliptic units (see [Ru4]).

The classical Euler Systems of Gauss sums (see [Ru5]) can be recovered very much in the same way, as a particular case of the situation where $k$ is totally real, $\mathcal{K}$ consists of CM abelian extensions $K$ of $k$, and appropriate choices for the sets $S_{K}$ containing a fixed number $r$ of non-archimedean primes of $k$ which split completely in $K / k$.

In the case where $\operatorname{char}(k)=p$ and $\operatorname{card}\left(S_{\infty}\right)=1$, the above construction leads to slight alterations of the Euler Systems of torsion points of rank one signnormalized Drinfeld Modules constructed in $[\mathbf{F X}]$.

However, in all the classical Euler system constructions enumerated above, the order of vanishing of the associated $L$-functions is 1 . If proved to be true, Rubin's 
Conjecture should be viewed as the source of non-trivial Euler Systems of units in the arbitrary order of vanishing case. It can be shown that the weaker conjecture $\mathrm{C}$ (see $\S 2.3$ above) has the same consequences in this context.

4.2. Groups of Special Units. Gras-type Conjectures. We work with the notations and under the assumptions of $\S 4.1$. We fix a field $K \in \mathcal{K}$. Our goal is to construct a group of special units $\mathcal{E}_{K, S_{\infty}, T}$ sitting inside $U_{K, S_{\infty}, T}$ as a $\mathbb{Z}[G(K / k)]-$ submodule of finite index and satisfying an analogue of the classical Gras Conjecture for the group of cyclotomic units of a real cyclotomic field $\mathbb{Q}\left(\zeta_{m}\right)^{+}$. We follow [P3], where the construction of $\mathcal{E}_{K, S_{\infty}, T}$ and proofs of the Gras Conjectures were given in detail in the function field context, as consequences of the fact that Rubin's Conjecture is true up to primes dividing the order of the Galois group $G(K / k)$ (see Corollary 3.1.9(1) above). The constructions and proofs given in [P3] carry over almost word for word to the present more general context. We remind the reader that our main assumption is that conjecture $B\left(L / k, S_{L}, T, r\right)$ holds true for all $L \in \mathcal{K}$.

Definition 4.2.1. Let $h_{k, S_{\infty}, T}:=\operatorname{card}\left(A_{k, S_{\infty}, T}\right)$. The group of special units $\mathcal{E}_{K, S_{\infty}, T}$ is defined to be the $\mathbb{Z}[G(K / k)]$-submodule of $U_{K, S_{\infty}, T}$ generated by

$$
u^{h_{k, S_{\infty}, T}}, \quad \eta_{K^{\prime}} \cdot \widetilde{\Phi}_{K^{\prime}}\left(\varepsilon_{K^{\prime}}\right),
$$

for all units $u \in U_{k, S_{\infty}, T}$, fields $K^{\prime} \in \mathcal{K}$ with $K^{\prime} \subseteq K, \eta_{K^{\prime}} \in I\left(G\left(K^{\prime} / k\right)\right)$, and $\Phi_{K^{\prime}} \in\left(U_{K^{\prime}}^{*}\right)^{(r-1)}$.

Proceeding as in [P3], one can eliminate $T$ from the constructions above and define what we call the group of Stark $S_{\infty}$-units of $K$ (or plainly Stark units, if $\operatorname{char}(K)=0)$ as follows.

Definition 4.2.2. The group of Stark $S_{\infty}$-units $\mathcal{E}_{K, S_{\infty}}$ of $K$ is defined to be the $\mathbb{Z}[G(K / k)]$-submodule of $U_{K, S_{\infty}}$ generated by

$$
\mu_{K}, \quad \mathcal{E}_{K, S_{\infty}, T},
$$

for all sets $T$ as above, such that $S_{\infty} \cap T=\emptyset$.

A direct application of Kolyvagin's Euler System techniques (see [Ru1], [Ru2] and $[\mathbf{R u 4}]$ ) relying on the fundamental fact that each of the generators of $\mathcal{E}_{K, S_{\infty}, T}$ which is not contained in the base field $k$ is the starting point of an Euler System of units (see §4.1) leads to a proof of parts (2) and (3) of the following result. The proof of part (1) is elementary (see [P3]).

TheOREM 4.2.3. If $\operatorname{char}(k)=0$ and conjecture $B\left(L / k, S_{L}, T, r\right)$ is true for all $L \in \mathcal{K}$, then

1. The indices $\left[U_{K, S_{\infty}, T}: \mathcal{E}_{K, S_{\infty}, T}\right]$ and $\left[U_{K, S_{\infty}}: \mathcal{E}_{K, S_{\infty}}\right]$ are finite.

2. For all $\ell$ prime, $\ell \chi \operatorname{card}(G(K / k))$, and all $\chi \in \widehat{G(K / k)}$, we have

$$
\operatorname{card}\left(\left(A_{K, S_{\infty}, T} \otimes \mathbb{Z}_{\ell}\right)^{\chi}\right)^{r}=\operatorname{card}\left(\left(U_{K, S_{\infty}, T} / \mathcal{E}_{K, S_{\infty}, T} \otimes \mathbb{Z}_{\ell}\right)^{\chi}\right) .
$$

3. For all $\ell$ prime, $\ell \chi \operatorname{card}(G(K / k)) \cdot \operatorname{card}\left(\mu_{K}\right)$, and all $\left.\chi \in \widehat{G(K / k}\right)$, we have

$$
\operatorname{card}\left(\left(A_{K, S_{\infty}} \otimes \mathbb{Z}_{\ell}\right)^{\chi}\right)^{r}=\operatorname{card}\left(\left(U_{K, S_{\infty}} / \mathcal{E}_{K, S_{\infty}} \otimes \mathbb{Z}_{\ell}\right)^{\chi}\right) .
$$


In the case $\operatorname{char}(k)=p$, one could try to prove the theorem above by employing the Euler System technique as well. Such an attempt would be successful with a single, but extremely important exception. In (2) and (3) one would be forced to assume that $\ell \neq p$, because in characteristic $p$ the Euler System technique fails to give upper bounds for the $p$-primary part of the Selmer group in question (which in the present setting is an ideal class-group). However, in [P3] we construct the groups of special units $\mathcal{E}_{K, S_{\infty}, T}$ and $\mathcal{E}_{K, S_{\infty}}$ in characteristic $p$ and prove the analogue of Theorem 4.2.3 above unconditionally and without making use of Kolyvagin's Euler System technique. In fact, we show that if conjecture $\mathbb{Z}\left[1 /\left|G\left(K^{\prime} / k\right)\right|\right] B\left(K^{\prime} / k, S^{\prime}, T^{\prime}, r^{\prime}\right)$ holds true, for all $K^{\prime} \subseteq K$ and all $S^{\prime}, T^{\prime}, r^{\prime}$, such that $\left(K^{\prime} / k, S^{\prime}, T^{\prime}, r^{\prime}\right)$ satisfies $\left(H_{r^{\prime}}\right)$, then the analogue of Theorem 4.2.3 follows. As conjecture $\mathbb{Z}\left[1 /\left|G\left(K^{\prime} / k\right)\right|\right] B\left(K^{\prime} / k, S^{\prime}, T^{\prime}, r^{\prime}\right)$ was proved in full generality in characteristic $p$ in $[\mathbf{P 2}]$ (see Corollary 3.1.9(1) above), we have the following (see [P3]).

THEOREM 4.2.4. If $\operatorname{char}(k)=p$, then

1. The indices $\left[U_{K, S_{\infty}, T}: \mathcal{E}_{K, S_{\infty}, T}\right]$ and $\left[U_{K, S_{\infty}}: \mathcal{E}_{K, S_{\infty}}\right]$ are finite.

2. For all $\ell$ prime, $\ell \chi \operatorname{card}(G(K / k))$, and all $\chi \in \widehat{G(K / k)}$, we have

$$
\operatorname{card}\left(\left(A_{K, S_{\infty}, T} \otimes \mathbb{Z}_{\ell}\right)^{\chi}\right)^{r}=\operatorname{card}\left(\left(U_{K, S_{\infty}, T} / \mathcal{E}_{K, S_{\infty}, T} \otimes \mathbb{Z}_{\ell}\right)^{\chi}\right) .
$$

3. For all $\ell$ prime, $\ell \chi \operatorname{card}(G(K / k)) \cdot \operatorname{card}\left(\mu_{K}\right)$, and all $\left.\chi \in \widehat{G(K / k}\right)$, we have

$$
\operatorname{card}\left(\left(A_{K, S_{\infty}} \otimes \mathbb{Z}_{\ell}\right)^{\chi}\right)^{r}=\operatorname{card}\left(\left(U_{K, S_{\infty}} / \mathcal{E}_{K, S_{\infty}} \otimes \mathbb{Z}_{\ell}\right)^{\chi}\right)
$$

In the particular case when $r=1$ (i.e. when $S_{\infty}$ consists of a single prime), we manage to improve (3) above slightly by proving the following (see $[\mathbf{P} 3]$ ).

TheOREM 4.2.5. If $\operatorname{char}(k)=p$ and $r:=\operatorname{card}\left(S_{\infty}\right)=1$, then

$$
\operatorname{card}\left(\left(A_{K, S_{\infty}} \otimes \mathbb{Z}_{\ell}\right)^{\chi}\right)=\operatorname{card}\left(\left(U_{K, S_{\infty}} / \mathcal{E}_{K, S_{\infty}} \otimes \mathbb{Z}_{\ell}\right)^{\chi}\right),
$$

for all prime numbers $\ell$, such that $\ell$ \ $\operatorname{card}(G(K / k))$.

Theorem 4.2.5 plays a crucial role in our proof of Chinburg's $\Omega_{3}$-conjecture for prime degree Galois extensions of function fields (see $[\mathbf{P 3}]$ ), a result also obtained by Bae earlier and with different methods (see [Bae]).

ExAmPles AND REmaRKS. In the case where $k=\mathbb{Q}$ and $K=\mathbb{Q}\left(\zeta_{m}\right)^{+}$(for which $S_{\infty}=\{\infty\}$, and therefore $r=1$ ), the group of Stark units $\mathcal{E}_{K, S_{\infty}}$ constructed above contains the classical group of cyclotomic units $\mathcal{C}_{m}$, constructed by Sinnott in $[\mathbf{S i}]$, with a finite, possibly non-trivial 2-power index. The reader can prove this statement without difficulty, as a consequence of the Remark at the end of $\S 4.1$. Therefore, if restricted to this case, Theorem 4.2.3(3) above leads to the classical Gras Conjecture.

THEOREM (the Gras Conjecture). Let $\ell$ be an odd prime number with the property that $\ell \chi \operatorname{card}\left(G\left(\mathbb{Q}\left(\zeta_{m}\right)^{+} / \mathbb{Q}\right)\right)$. Then, for all $\chi \in G\left(\widehat{\mathbb{Q}\left(\zeta_{m}\right)^{+}} / \mathbb{Q}\right)$, we have

$$
\operatorname{card}\left(\left(A_{m} \otimes \mathbb{Z}_{\ell}\right)^{\chi}\right)=\operatorname{card}\left(\left(U_{m} / \mathcal{C}_{m} \otimes \mathbb{Z}_{\ell}\right)^{\chi}\right)
$$

where $A_{m}$ and $U_{m}$ are the ideal-class group and the group of global units of $\mathbb{Q}\left(\zeta_{m}\right)^{+}$.

The theorem above was proved first by Mazur and Wiles in $[\mathbf{M W}]$, as a consequence of their proof of the Iwasawa Main Conjecture over $\mathbb{Q}$, and following 
Greenberg's earlier insightful remarks $[\mathbf{G r 1}],[\mathbf{G r 2}]$. Later, Kolyvagin and Rubin (see for example [Ru2]) gave a different proof, based on Kolyvagin's Euler System techniques applied to the particular case of Euler Systems of cyclotomic units.

The groups of special units $\mathcal{E}_{K, S_{\infty}}$ constructed above should be viewed as generalizations of Sinnott's groups of cyclotomic units $\mathcal{C}_{K}$ (if $k=\mathbb{Q}$ ), elliptic units (if $k$ is a quadratic imaginary field), or Hayes elliptic units [H4] (if $k$ is a function field). Theorems 4.2.3, 4.2.4, and 4.2.5 should be regarded as generalizations of the classical Gras Conjecture stated above.

\section{References}

[A1] Anderson, G., t-motives, Duke Math. Jour. 53 (1986), 457-502.

[A2] Anderson, G., Another look at the index formulas of cyclotomic number theory, Journal of Number Theory 60 (1996), 142-164.

[Bae] Bae, S., On the conjecture of Lichtenbaum and of Chinburg over function fields, Math. Ann. 285, no. 3 (1989), 417-445.

[Bar1] Barsky, D., Fonctions zêta p-adiques d'une classe de rayon des corps de nombres totalement réels, Groupe d'Etude d'Analyse Ultramétrique (1977/78), Exp. No. 16.

[Bar2] Barsky, D., Sur la nullité du $\mu$-invariant analytique d'Iwasawa pour les fonctions L $p$ adiques des corps totallement reéls, (preprint - November 20, 2003).

[BK] Bloch, S. and Kato, K., L-functions and Tamagawa numbers of motives, In The Grothendieck Festschrift, Progr. in Math. 86, Birkhäuser, Boston 1 (1990), 333-400.

[Br] Brumer, A., On the units of algebraic number fields, Mathematika 14 (1967), 121-124.

[Bu1] Burns, D., Equivariant Tamagawa numbers and refined abelian Stark Conjectures, J. Math. Sci., Tokyo 10 (2003), 225-259.

[Bu2] Burns, D., Iwasawa theory and p-adic Hodge theory over non-commutative algebras, Parts I and II, (preprints) (1997).

[Bu3] Burns, D., Equivariant Tamagawa numbers and Galois module theory I, Comp. Math. 129 (2001), 201-237.

[Bu4] Burns, D., On values of equivariant Zeta-functions of curves over finite fields (Very Preliminary Notes), (preprint) (2000).

[BF1] Burns, D. and Flach, M., Motivic L-functions and Galois module structure, Math. Ann. 305 (1996), 65-102.

[BF2] Burns, D. and Flach, M., On Galois structure invariants associated to Tate motives, Amer. J. Math. 120.

[BF3] Burns, D. and Flach, M., Equivariant Tamagawa numbers for motives, (preprint) (1998).

[CaN] Cassou-Noguès, P., Valeurs aux entiers négatifs des fonctions zêta et fonctions zêta $p$ adiques, Invent. Math. 51, no. 1 (1979), 29-59.

[Ch1] Chinburg, T., On the Galois structure of algebraic integers and S-units, Invent. Math $\mathbf{7 4}$ (1983), 321-349.

[Ch2] Chinburg, T, Exact sequences and Galois module structure, Ann. of Math. 121 (1985), $351-376$.

[Co] Coates, J., p-adic L-functions and Iwasawa's theory, Algebraic number fields: L-functions and Galois properties (Proc. Sympos., Univ. Durham, Durham, 1975), Academic Press, London, 1977, pp. 269-353.

[DR] Deligne, P. and Ribet, K., Values of abelian L-functions at negative integers over totally real fields, Invent. Math. 59 (1980), 227-286.

[DST] Dummit, D., Sands, J., Tangedal, B., Stark's conjecture in multi-quadratic extensions revisited, (preprint).

[FW] Ferrero, B., Washington, L., The Iwasawa invariant $\mu_{p}$ vanishes for abelian number fields, Ann. of Math. (2) 109 (1979), 377-395.

[FX] Feng, K., Xu, F., Kolyvagin's "Euler systems" in cyclotomic function fields, J. Number Theory 57, no. 1 (1996), 114-121.

[Gra] Grant, D., Units from 5-torsion on the Jacobian of $y^{2}=x^{5}+1 / 4$ and the conjectures of Stark and Rubin, (preprint).

[Gr1] Greenberg, R., On p-adic L-functions and cyclotomic fields, Nagoya Math. J. 56 (1975), 61-77. 
[Gr2] Greenberg, R., On p-adic L-functions and cyclotomic fields. II. Nagoya Math. J. 67 (1977), 139-158.

[Gre1] Greither, C., The structure of some minus class groups and Chinburg's third conjecture for abelian fields, Math. Z. 229, no. 1 (1998), 107-136.

[Gre2] Greither, C., Some cases of Brumer's conjecture for abelian CM extensions of totally real fields, Math. Z. 233, no. 2 (2000), 515-534.

[Gro1] Gross, B.H., $p$-adic L-series at $s=0$, J.Fac.Sci.Univ. Tokyo 28 (1981), 979-994.

[Gro2] Gross, B.H., On the values of abelian L-functions at $s=0$, J.Fac.Sci.Univ. Tokyo 35 (1988), 177-197.

[Gro3] Gross, B.H., Letter to John Tate, November 1993.

[GRW] Gruenberg, K.W., Ritter, J. and Weiss, A., A local approach to Chinburg's Root Number Conjecture, to appear in Proc. London Math. Soc..

[GS] Goss, D. and Sinnott, W., Class-groups of function fields, Duke Math. J. 52 (1985), 507-516.

[H1] Hayes, D.R., Stickelberger elements in function fields, Comp. Math. 55 (1985), 209-239.

[H2] Hayes, D.R., The refined $\wp$-adic abelian Stark conjecture in function fields, Inv. Math. 94 (1988), 505-527.

[H3] Hayes, D.R., Base change for the conjecture of Brumer-Stark, J. reine angew. Math 497 (1998), 83-89.

[H4] Hayes, D.R., Elliptic units in function fields, Number theory related to Fermat's last theorem (Cambridge, Mass., 1981) 26 (1982), Progr. Math., Birkhuser, Boston, Mass., 321-340.

[Ka1] Kato, K., Iwasawa theory and p-adic Hodge theory, Kodai Math. J. 16 (1993), 1-31.

[Ka2] Kato, K., Lectures on the approach to Iwasawa theory for Hasse-Weil L-functions via $B_{d r}$, Part I, Arithmetical Algebraic Geometry (ed. E. Ballico), Springer LNM 1553 (1993), 50-163.

[Ki] Kisilevsky, H, Multiplicative independence in function fields, Journal of Number Theory 44 (1993), 352-355.

[KM] Knudsen, F. and Mumford, D., The projectivity of the moduli space of stable curves I: Preliminaries on 'det' and 'div', Math. Scand. 39 (1976), 19-55.

[Ku] Kurihara, M., Iwasawa theory and Fitting ideals, J. Reine Angew. Math. 561 (2003), 39-86.

[MW] Mazur, B. and Wiles, A., Class fields of abelian extensions of $\mathbb{Q}$, Invent. Math. 76 (1984), 179-330.

[P1] Popescu, C.D., On a refined Stark conjecture for function fields (1996), Ph.D. Thesis, Ohio State University.

[P2] Popescu, C.D., On a refined Stark conjecture for function fields, Comp. Math. 116 (1999), 321-367.

[P3] Popescu, C.D., Gras-type conjectures for function fields, Comp. Math. 118 (1999), no. 3, 263-290.

[P4] Popescu, C.D., Base change for Stark-type conjectures "over $\mathbb{Z}$ ", J. Reine Angew. Math 542 (2002), 85-111.

[P5] Popescu, C.D., Stark's Question and a strong form of Brumer's conjecture extrapolated to function fields, to appear in Compositio Math. (2004).

[P6] Popescu, C.D., On the Rubin-Stark Conjecture for a special class of CM extensions of totally real number fields, to appear in Math. Zeitschr. (2004).

[P7] Popescu, C.D., The Rubin-Stark Conjecture for imaginary abelian fields of odd prime power conductor, to appear in Math. Annalen (2004).

[P8] Popescu, C.D., The Rubin-Stark Conjecture for a class of extensions of function fields, in preparation.

[P9] Popescu, C.D., On the Rubin-Stark Conjecture over $\mathbb{Q}$, in preparation.

[P10] Popescu, C.D., 1-Motives and Iwasawa Theory, in preparation.

[P11] Popescu, C.D., On the Brumer-Stark Conjecture and its generalizations over arbitrary totally real number fields, in preparation.

[P12] Popescu, C.D., Gross-type $\ell$-adic refinements of the Rubin-Stark conjecture, in preparation.

[PR] Perrin-Riou, B., Fonctions L p-adiques des représentations p-adiques, Astérisque 229. 
[R] Rapoport, M. et al., Editors, Beilinson's Conjectures on Special Values of L-functions, Perspectives in Math. 4 (1987), Academic Press.

[Ru1] Rubin, K., The main conjecture, Appendix of S. Lang's Cyclotomic Fields I and II, Springer Verlag (1990).

[Ru2] Rubin, K., Stark units and Kolyvagin's "Euler systems", J. Reine Angew. Math. 425 (1992), 141-154.

[Ru3] Rubin, K., A Stark conjecture "over $\mathbb{Z}$ " for abelian L-functions with multiple zeros, Annales de L'Institut Fourier 46 (1996), 33-62.

[Ru4] Rubin, K., Euler Sytems, Annals of Mathematics Studies 147 (2000), Hermann Weyl Lectures. The Institute for Advanced Study. Princeton University Press, Princeton, NJ.

[Ru5] Rubin, K., Kolyvagin's system of Gauss sums., Arithmetic algebraic geometry (Texel, 1989) 89 (1991), Progr. Math., Birkhuser Boston, Boston, MA, 309-324.

[Sa1] Sands, J.W., Abelian fields and the Brumer-Stark conjecture, Comp. Math. 43 (1984), $337-347$.

[Sa2] Sands, J.W., Galois groups of exponent two and the Brumer-Stark conjecture, J. Reine Angew. Math. 349 (1984), 129-135.

[Sa3] Sands, J.W., Stark's question and Popescu's conjecture for abelian L-functions, Number Theory (Turku, 1999) (2001), de Gruyter, Berlin, 305-315.

[Sch1] Schmidt, A., An arithmetic site for the rings of integers of algebraic number fields, Inv. Math. 123 (1996), 575-610.

[Sch2] Schmidt, A., On p-adic zeta-functions associated to the positive topology of algebraic number fields, Publ. RIMS, Kyoto Univ. 33 (1997), 151-161.

[Si] Sinnott, W., On the Stickelberger ideal and the circular units of a cyclotomic field, Ann. of Math. 108 (1978), 107-134.

[St] Stark, H., L-functions at $s=1, I, I I, I I I, I V$, Adv. in Math. 7 (1971), 301-343; 17 (1975), 60-92; 22 (1976), 64-84; 35 (1980), 197-235.

[T] Tan, K.S., Generalized Stark formulae over function fields, preprint.

[Tgd] Tangedal, B., The conjectures of Sands and Stark for special values of abelian L-functions, Ph.D. Thesis, U. C. San Diego (1994).

[Ta1] Tate, J., The cohomology groups of tori in finite Galois extensions of number fields, Nagoya Math. J. 27 (1966), 709-719.

[Ta2] Tate, J., Brumer-Stark-Stickelberger, Séminaire de Théorie des Nombres, Univ. de Bordeaux (1980-1981), exp. 24.

[Ta3] Tate, J., On Stark's conjecture on the behaviour of $L(s, \chi)$ at $s=0$, J. Fac. Sci. Univ. Tokyo 28 (1982), 963-978.

[Ta4] Tate, J., Les conjectures de Stark sur les fonctiones L d'Artin en $s=0$, Progr. in Math. 47 (1984), Boston Birkhäuser.

[Ta5] Tate, J., Private Communication (1997-2000).

[Wi1] Wiles, A., The Iwasawa conjecture for totally real fields, Ann. of Math. 131 (1990), 493-540.

[Wi2] Wiles, A., On a conjecture of Brumer, Ann. of Math. 131 (1990), 555-565.

[Y] Yang, T-H, Existence of algebraic Hecke characters, C. R. Acad. Sci. Paris Sr. I Math. 332, no. 12 (2001), 1041-1046.

Department of Mathematics, University of California at San Diego, La Jolla, CA 92093-0112

E-mail address: cpopescu@math.ucsd.edu 